$\sqrt{67}$ thrive IGGAPH2019

ADVANCED USE CASES FOR ANIMATION RIGGING IN UNITY

SIGGRAPH Studio Workshop

Dave Hunt, Technical Artist, Unity

Olivier Dionne, Animation Developer, Unity

Simon Bouvier-Zappa, Animation Developer, Unity
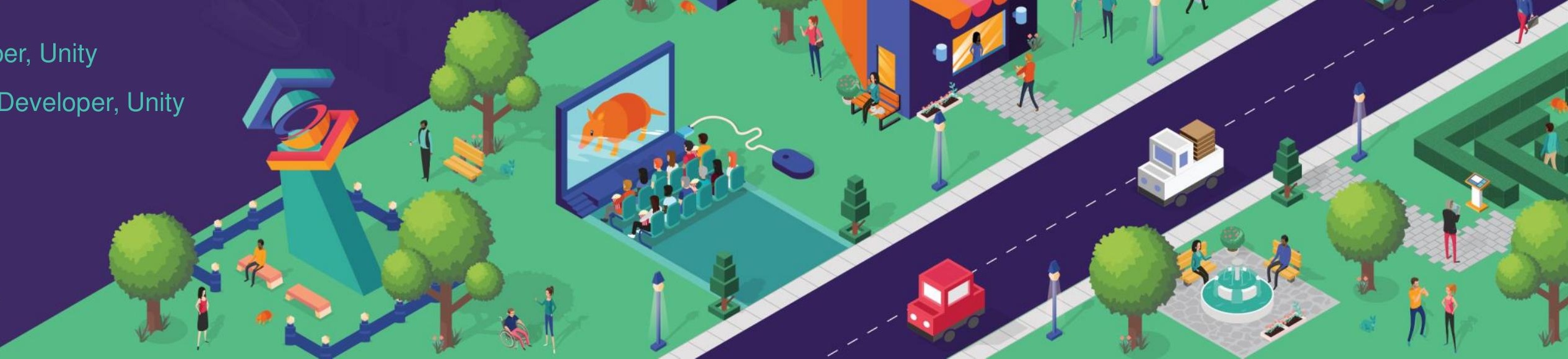


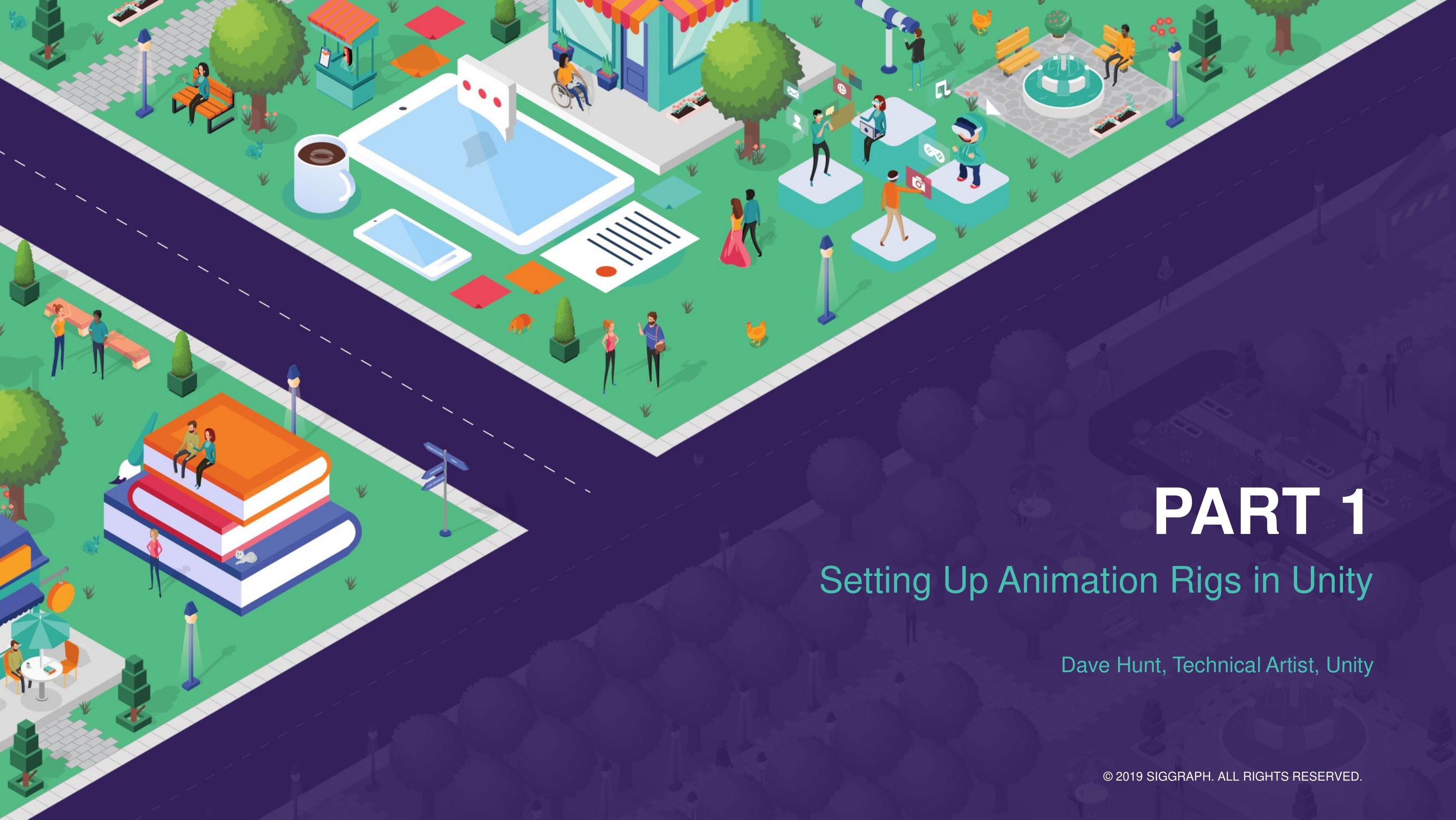




\section{INTRODUCTION TO THE ANIMATION RIGGING PACKAGE}

\section{- Overview}

- The Animation Rigging (Preview) package enables procedural motion for animated skeletons at runtime

- Unity 2019.1 - Runtime Rigging

- Unity 2019.2 - Keyframing in Animation Window and Effectors - Enables motion editing workflows in the Unity Editor

- Unity 2019.3 - Keyframing in Timeline

- Enables multi-track, layered animation authoring

- Installation

- One-click installation from Package Manager

- Samples and Documentation

- Links are included in Package Manager

Online docs:

https://docs.unity3d.com/Packages/com.unity.animation.rigging @0.2/manual/index.html
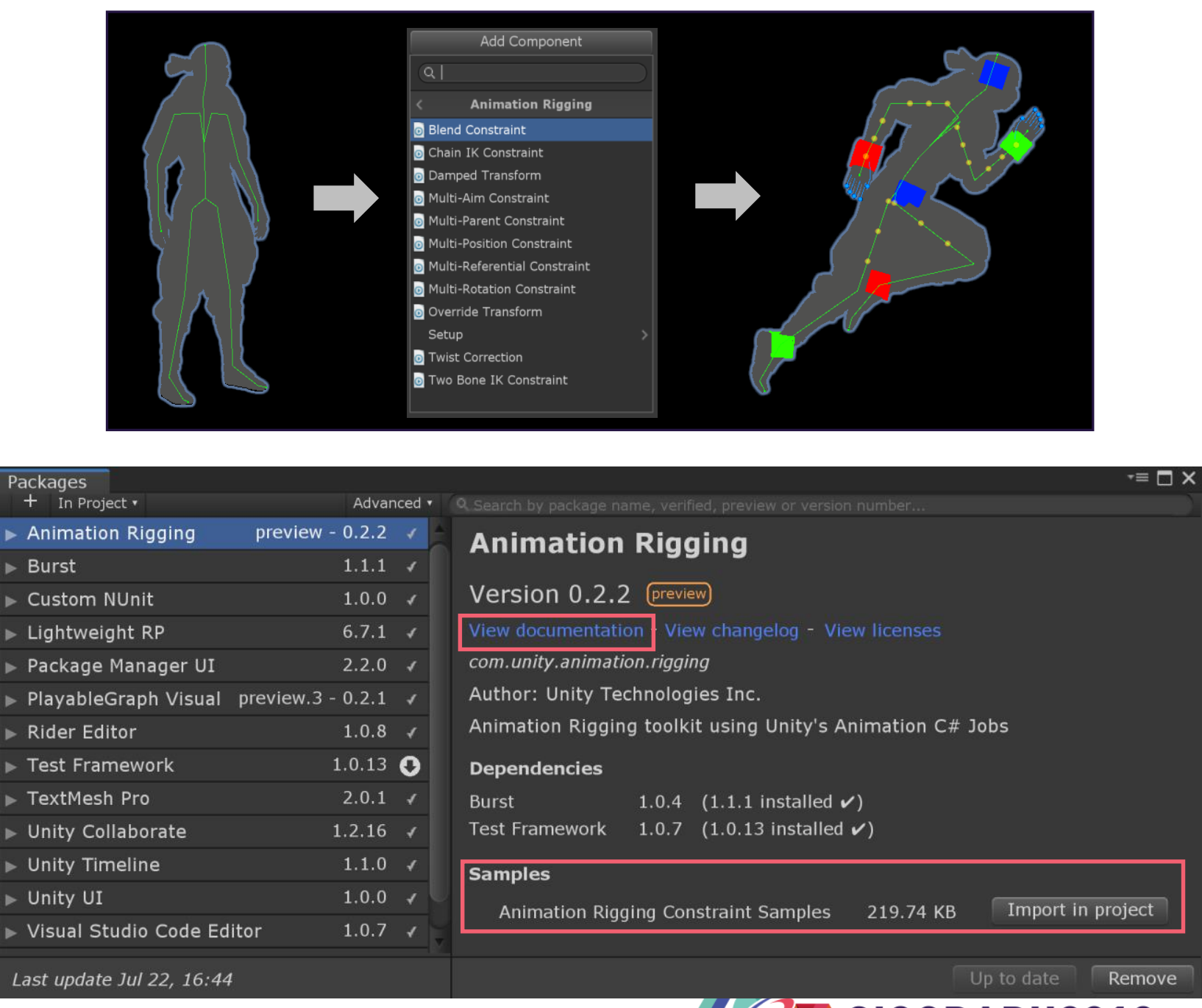


\section{WORKSHOP: SETTING UP A FULL BODY CHARACTER RIG}

- This rig works for Runtime Rigging and Animation Authoring

- All characters are playing the same animation clip

- Animation variations are made using keyframed rig overrides
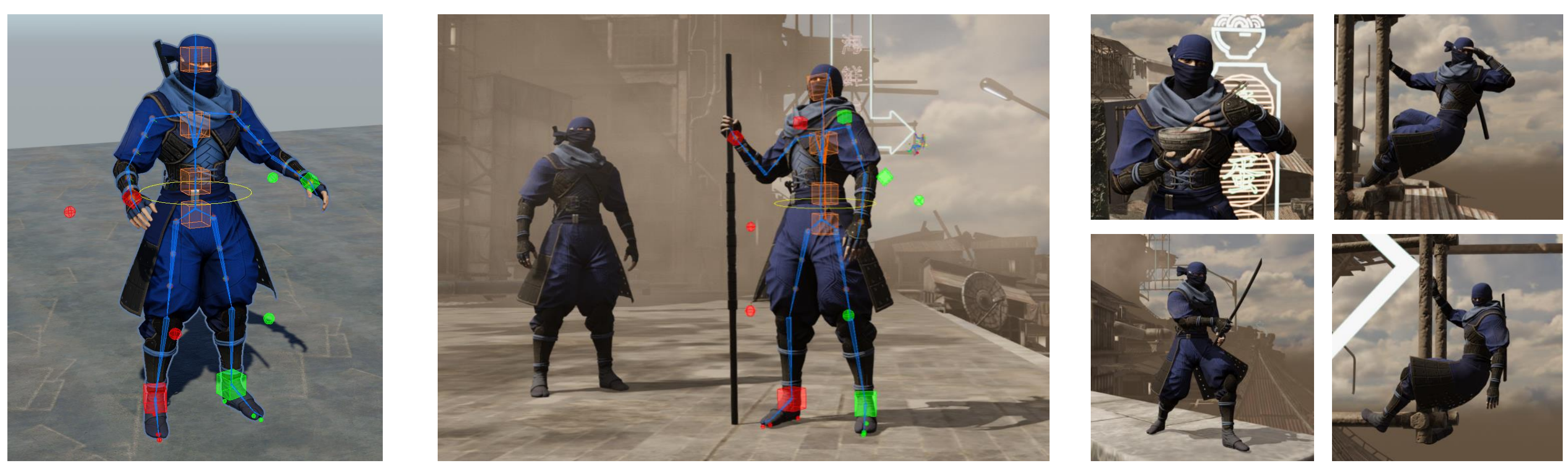


\section{SETTING UP THE SKELETON}

Open the scene: 01_WorkshopStart

- Bone Renderer

- See and interact with skeletons in the Scene

- Multiple Bone Renderer components have already been added to the Animator Root

- One for the main body skeleton

- Another for the twist bones

- Setup instructions (this is already setup)

1. Add Bone Renderer component

2. Add transforms to the list

3. Customize display options

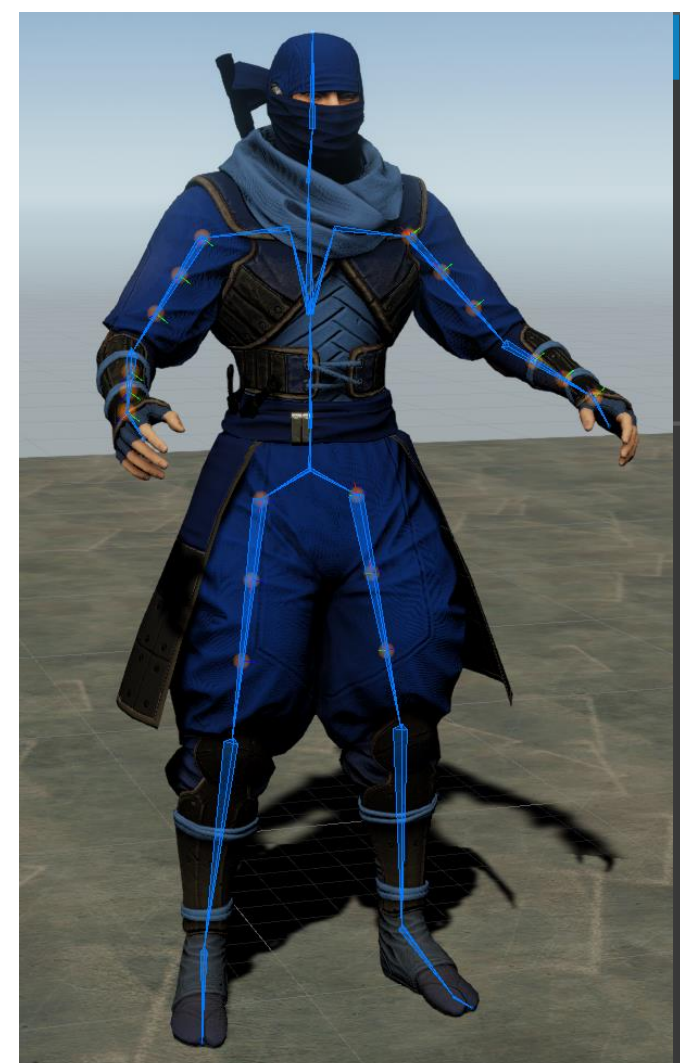

\section{$\square$ Bon
Bone Size \\ Shape \\ Color}

Tripod Size

Transforms

[ $\checkmark$ Bone Renderer (Script)

Bone Size

Shape

Color

Tripod Size

$\checkmark$ Transforms
Size
Element 0
Element 1
Element 2

|

0.7

$\checkmark$

Pyramid
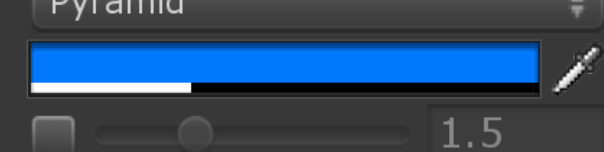

1.5

[7]

2.5

$\checkmark$

Pyramid

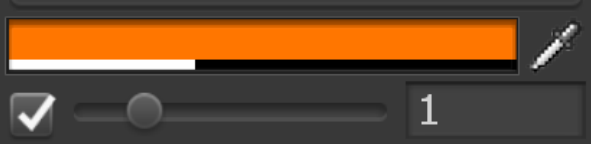

18

RightLowerArmTwist1

RightLowerArmTwist2

RightLowerArmTwist3 


\section{SETTING UP A CONTROL RIG FOR THE FULL BODY}

- Organizing the rig in Regions

- Arms

- Legs

- Spine (with head)

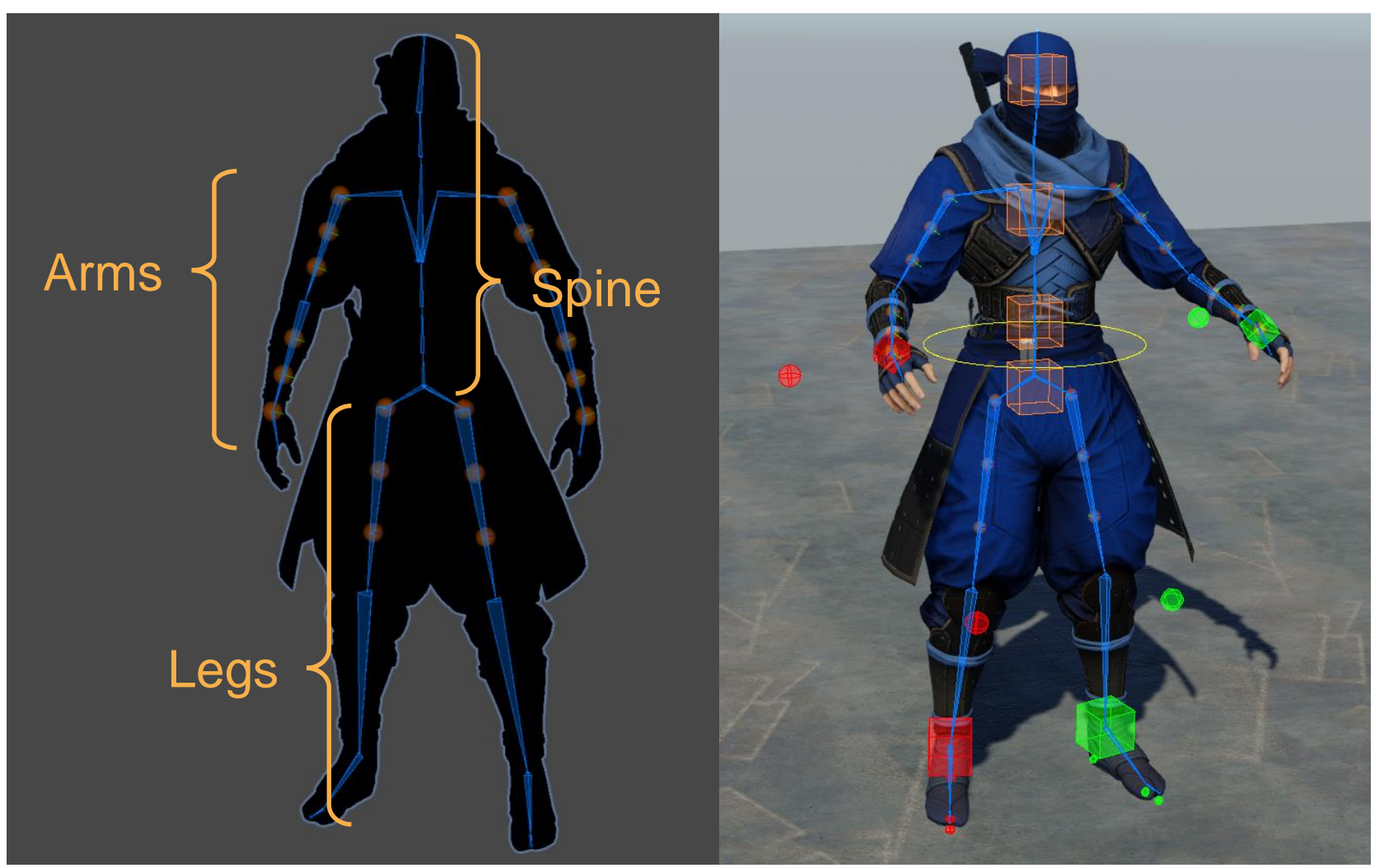




\section{SETTING UP A CONTROL RIG FOR THE FULL BODY}

- Create a new Rig for the control rig

- There can be multiple rigs!

- Setup instructions

1. Select NinjaRig and add the Rig Builder component

1. In the Rig Layers list click the + button to add a new entry

2. Create a new child GameObject and name it ControlRig

1. Add the Rig component

3. Drag and drop ControlRig into the Rig Layers list

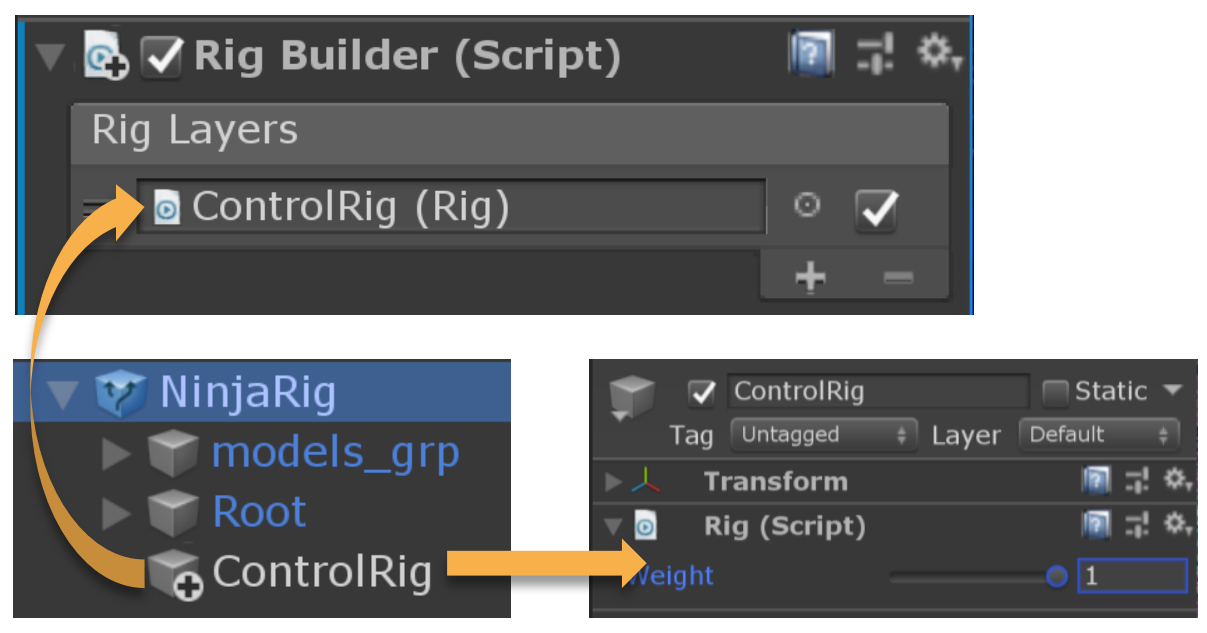




\section{SETTING UP THE ARM REGION - RIGHT SIDE}

- Create the Right Arm region

\section{- Setup instructions}

1. Create a new GameObject below ControlRig, name it "RightArm"

2. Add the TwoBonelKConstraint component

3. Add the arm bones for Root, Mid and Tip (see screenshot)

- Note: it is helpful to lock the Inspector while adding scene objects

O Inspector

- $=$

4. Add Effectors

1. Create two new GameObjects below RightArm and name them "RightArm_target" and "RightArm_hint"

2. Assign these to the TwoBonelK - Target and Hint fields

3. Customize the effector display options

4. Align the effectors to the skeleton

1. Select RightArm_target, then ctrl-select the RightHand bone

2. On the Animation Rigging menu click Align Transform

3. Also align the RightArm_hint to the RightLowerArm bone

5. Press Play $\square$ to see and interact with the Arm IK rig

6. Or press Preview in Animation Window
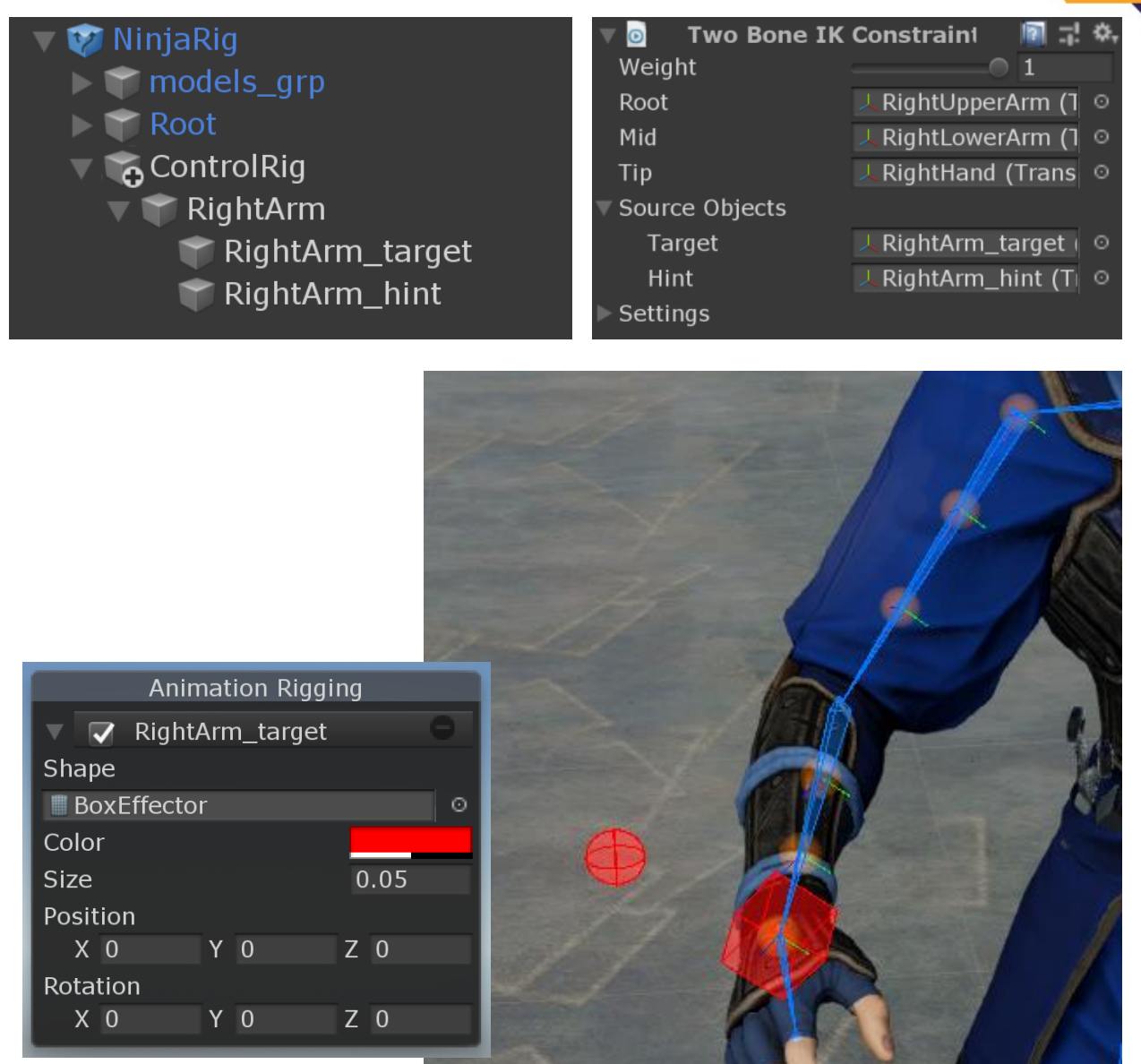

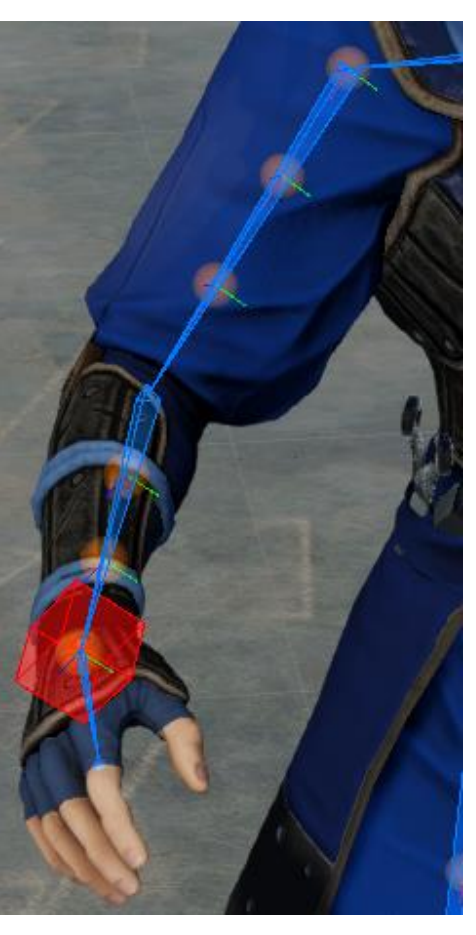

thrive

SIGGRAPH2019 


\section{SETTING UP THE ARM REGION - LEFT SIDE}

- Create the Left Arm region (auto-setup)

- Automation script example:

- Assets/Editor/TwoBonelKAutoSetup.cs

- Setup instructions

1. Create a new GameObject below ControlRig, name it "LeftArm"

2. Add the TwoBonelKConstraint component

3. Add only the Tip bone

4. With the TwoBonelK object selected, run the auto-setup command... 1. Animation Rigging $>$ Utilities $>$ Auto-Setup TwoBonelK from Tip Transform

5. Customize the Effector display options

Auto-Setup TwoBonelK from Tip Transform

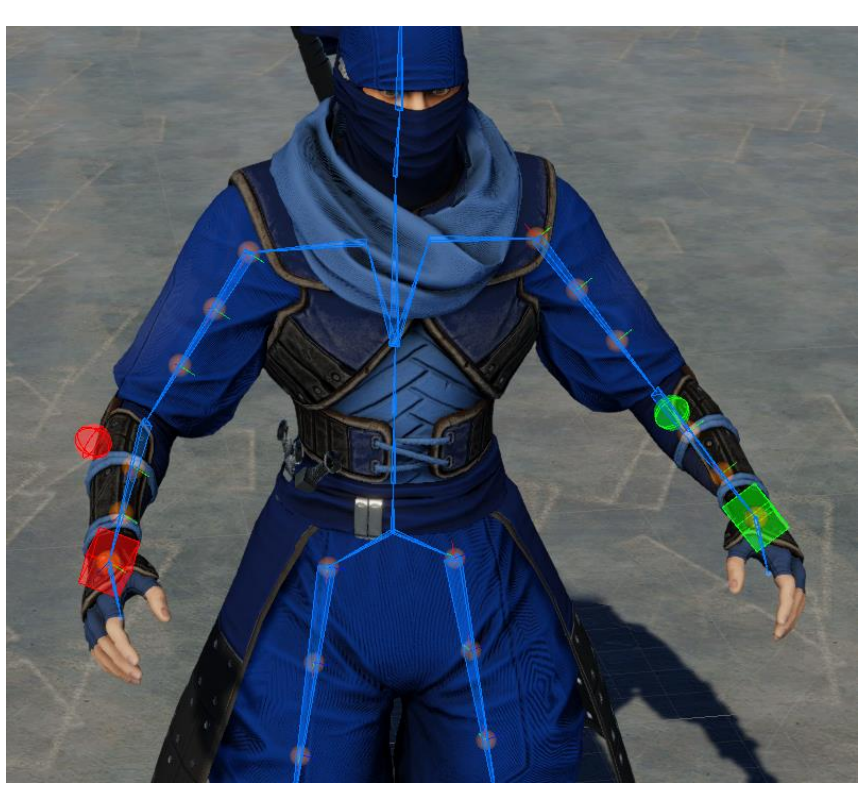




\section{SETTING UP THE LEG REGION - RIGHT SIDE}

- Create the Right Leg region

- TwoBonelK with remote pivots using Multi-Referential Constraints

\section{- Setup instructions}

1. Create a GameObject for the leg IK (child of ControlRig, named "RightLeg")

1. Add a TwoBonelK component

2. Assign the RightFoot bone as Tip and run Auto-Setup TwoBonelK

- Animation Rigging > Utilities > Auto-Setup TwoBonelK from Tip Transform

2. Add remote pivots for the foot

1. Create a group GameObject (child of RightLeg named "RemotePivots")

2. Create child GameObjects for: ToePivot, BallPivot and HeelPivot

3. Customize the Effector display options and align them to the character model

3. Add a Multi-Referential Constraint to the RightLeg

1. Assign the RightLeg_target and remote pivots as Reference Objects

2. ${ }^{* * *}$ Reorder the components so that Multi-Referential comes before TwoBonelK

4. Press Play and interact with the Multi-Referential Constraint

1. Change the Driving object to switch pivots

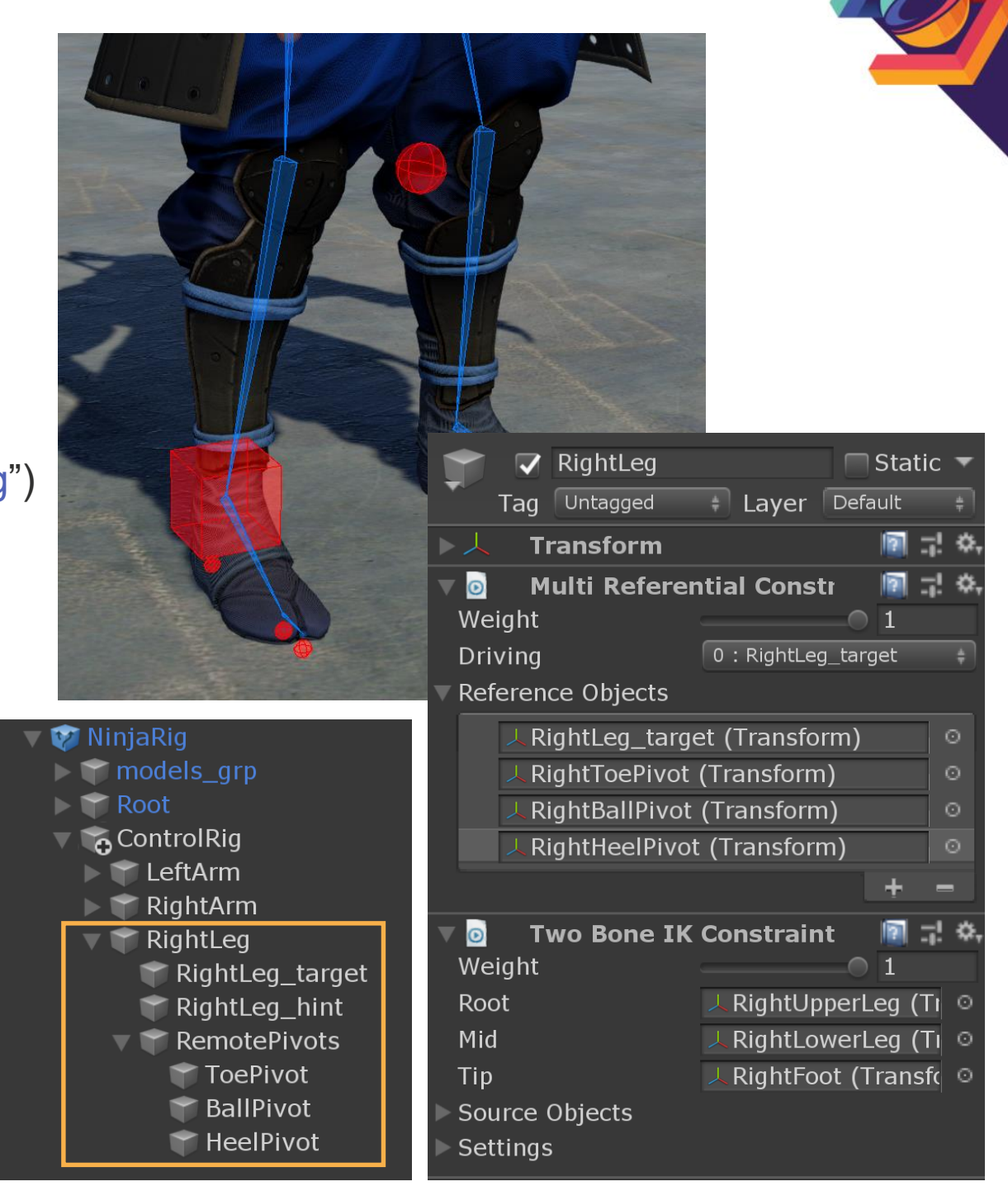




\section{SETTING UP THE LEG REGION - LEFT SIDE}

- Copy the Right Leg to the Left side using Prefabs

- The Prefab system makes it easy to save copies of rig regions. These can be shared with the same skeleton or different ones.

\section{- Setup instructions}

1. Save a prefab of the right leg

1. Drag and drop the RightLeg into a folder in your Project

2. Add a copy of the prefab for the left leg

1. Drag the RightLeg prefab onto ControlRig and rename it to LeftLeg

2. Assign the TwoBonelK Tip transform and run Auto-Setup TwoBonelK

3. Mirror the remote pivot Effector positions

1. Animation Rigging menu $>$ Utilities $>$ Mirror Transforms

2. Customize the Effector display options
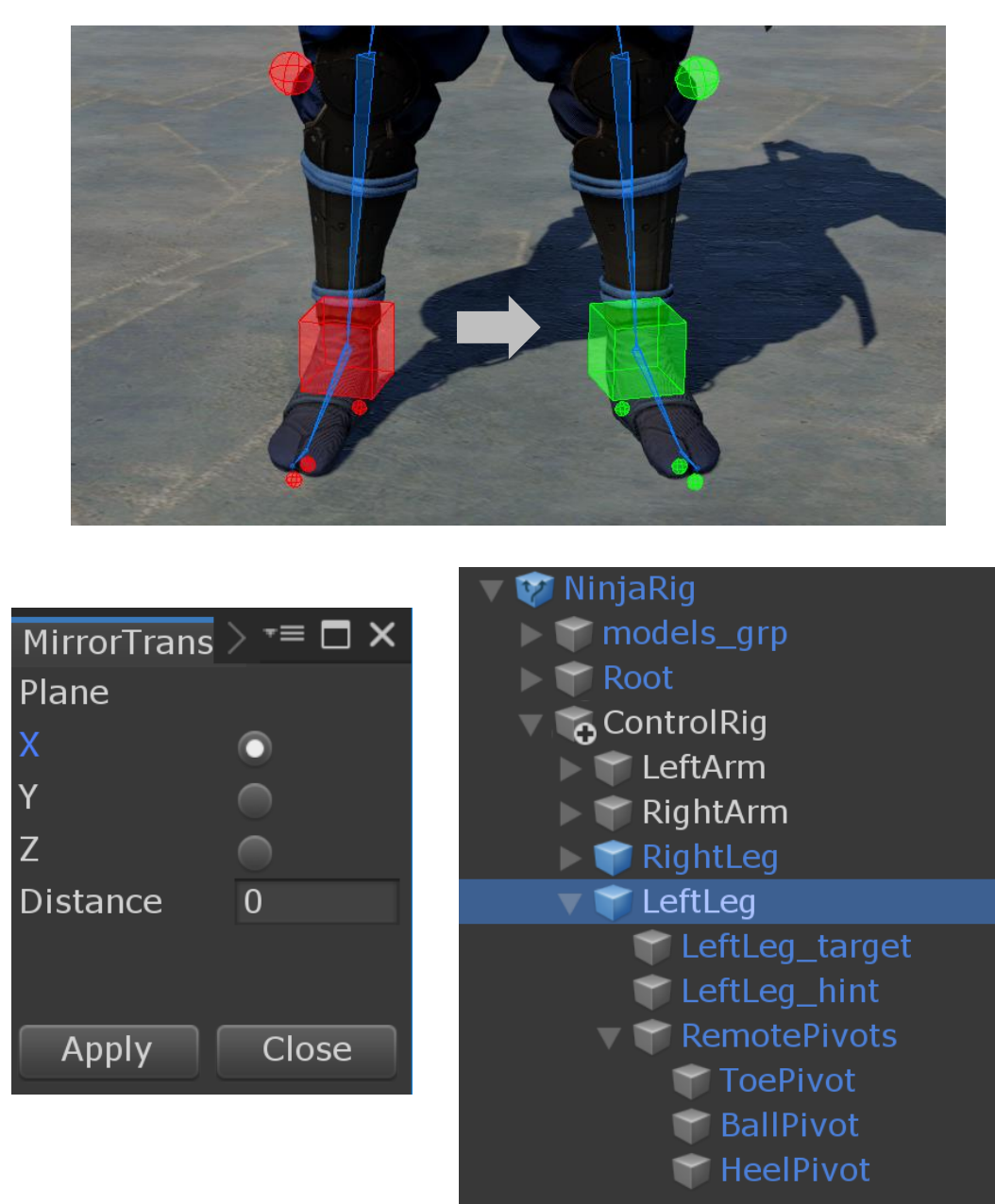


\section{SETTING UP THE SPINE REGION}

- Center of Gravity

- Top-level control for the whole spine

- Pelvis

- Independent rotation without affecting the upper body

- Torso

- Top and bottom rotation controls

- Automatic blended rotation of the middle bone(s)

- Head

- Single rotation control for the head

- Automatic blended rotation of the neck

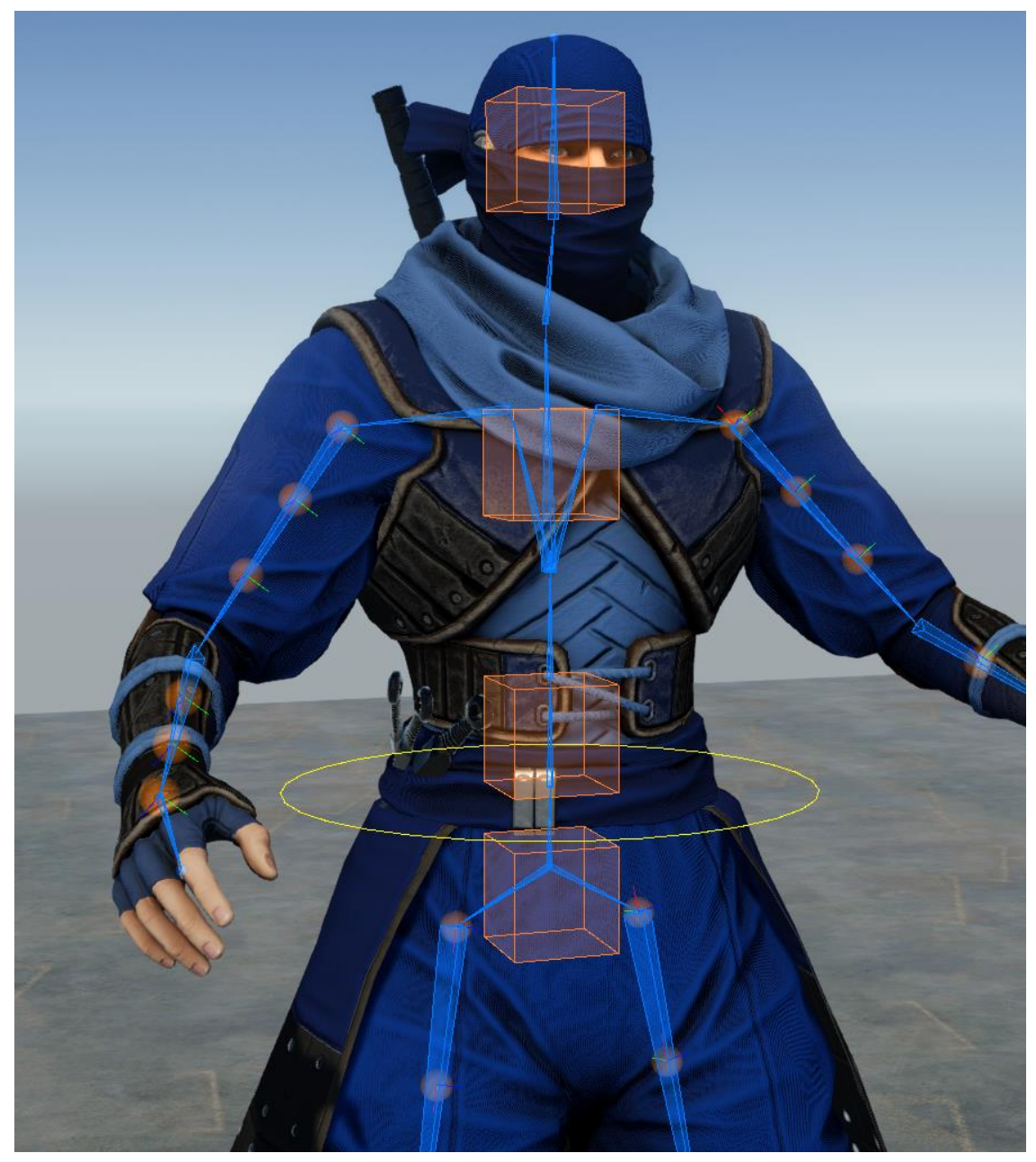




\section{SETTING UP THE SPINE REGION - TORSO AND HEAD}

Want to have:

- Torso

- Top and bottom rotation controls

- Automatically blend rotation of the middle bones

- Head

- Head rotation control with automatic blended neck rotation

- Let's develop a new constraint in C\#

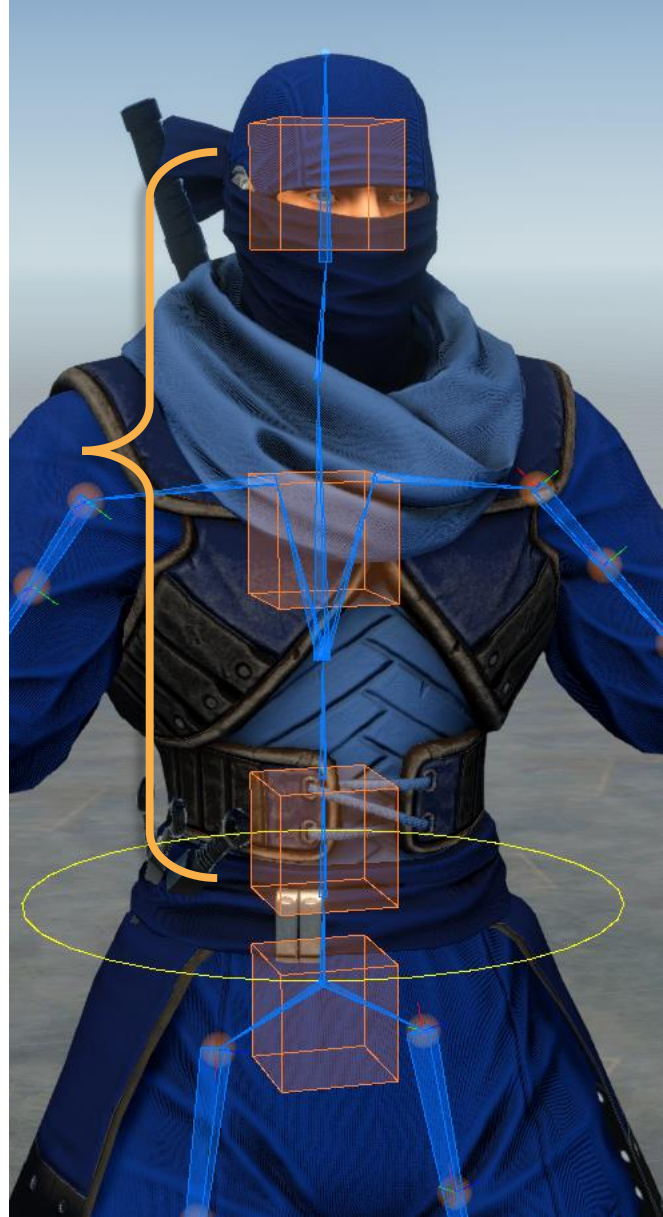


LOS ANGELES - 28 JULY - 1 AUGUST

\section{PART 2}

Extending the Animation Rigging package with C\#

Olivier Dionne, Animation Developer, Unity

Simon Bouvier-Zappa, Animation Developer, Unity 


\section{OPEN RIGGING TOOLKIT}

Each production has different requirements

- Custom constraints can be tailor-made to your specific use cases

- Potential performance gains

- Unique interactions or behaviors

Source of all constraints shipping with the package is available and thus editable/extensible 


\section{ANIMATION RIGGING PACKAGE IN A NUTSHELL}

- Built on top of Animation C\# jobs

- Blog post : https://blogs.unity3d.com/2018/08/27/animation-c-jobs/

- Enables you to modify an animation pose on the Animator thread prior to writing it back to the GameObjects

- Frictionless multi-threaded scheduling performed by the Animator

- Abstracts complexity required to create distributed constraints

- Interactive layering of multiple rigs and constraint combinations

WYSIWYG constraint scheduling via the hierarchy view 


\section{SCRIPTING A CUSTOM CONSTRAINT}

Boils down to declaring a RigConstraint

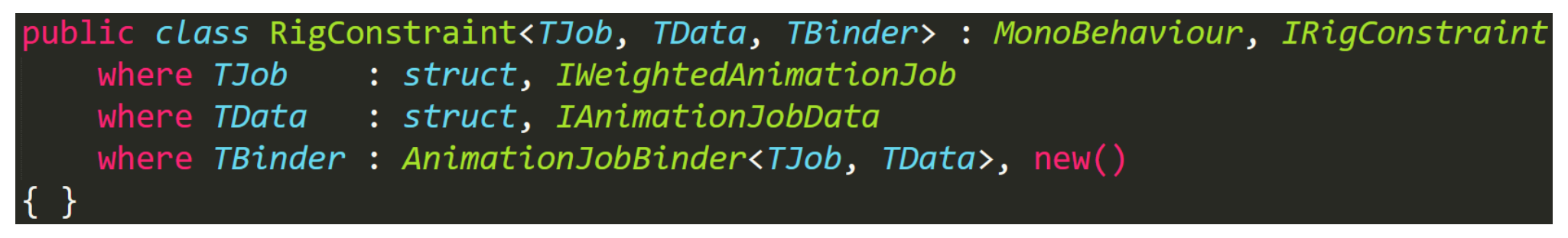




\section{SCRIPTING A CUSTOM CONSTRAINT}

Boils down to declaring a RigConstraint

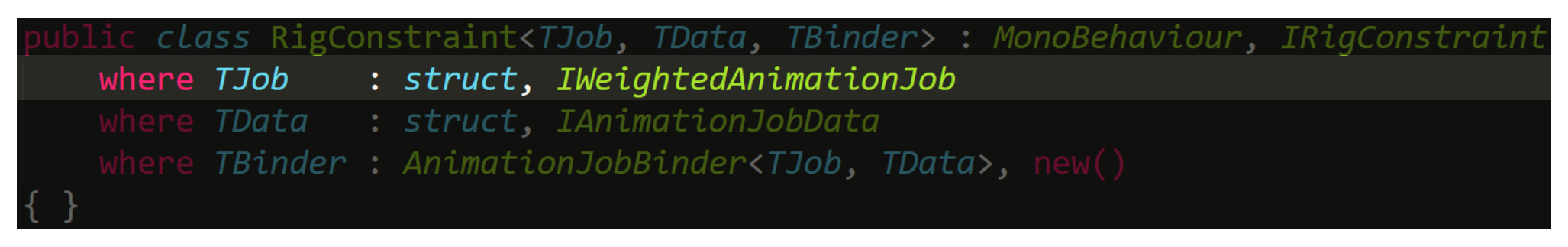




\section{SCRIPTING A CUSTOM CONSTRAINT}

Boils down to declaring a RigConstraint

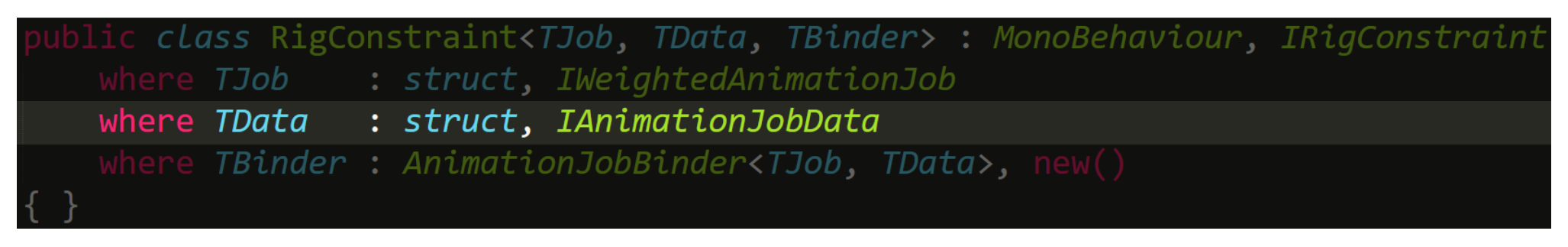




\section{SCRIPTING A CUSTOM CONSTRAINT}

Boils down to declaring a RigConstraint

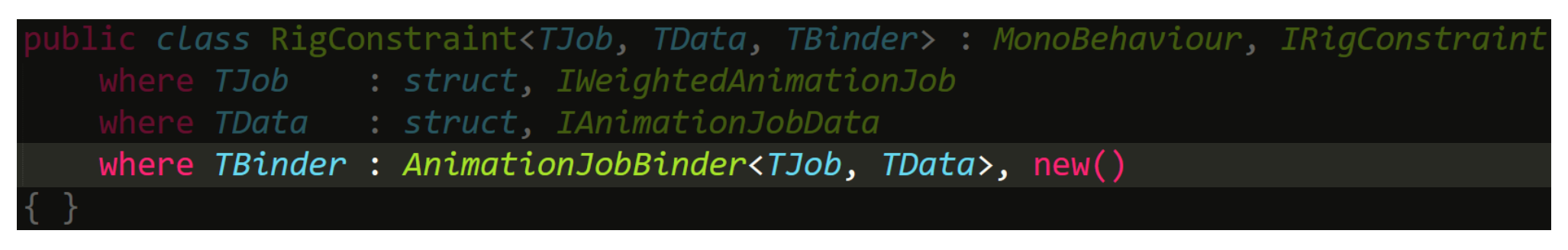




\section{SCRIPTING A CUSTOM CONSTRAINT}

Boils down to declaring a RigConstraint

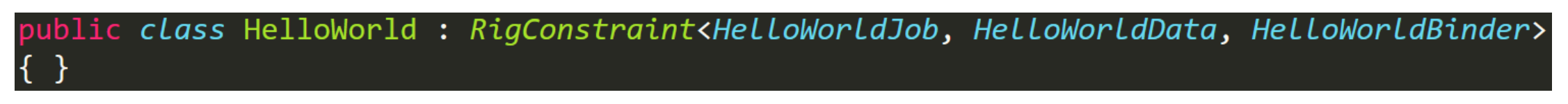




\section{SCRIPTING A CUSTOM CONSTRAINT}

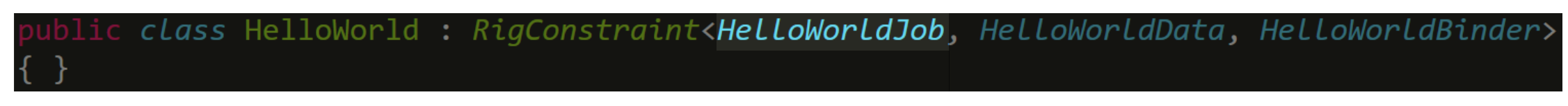

HelloWorldJob is an IWeightedAnimationJob

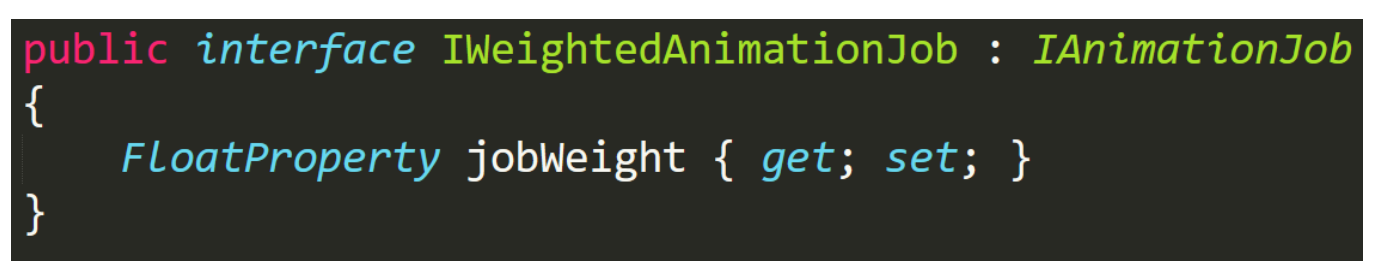

An IAnimationJob containing an auto populated weight property 


\section{SCRIPTING A CUSTOM CONSTRAINT}

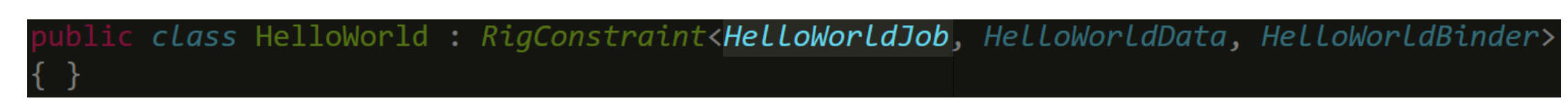

HelloWorldJob is an IWeightedAnimationJob

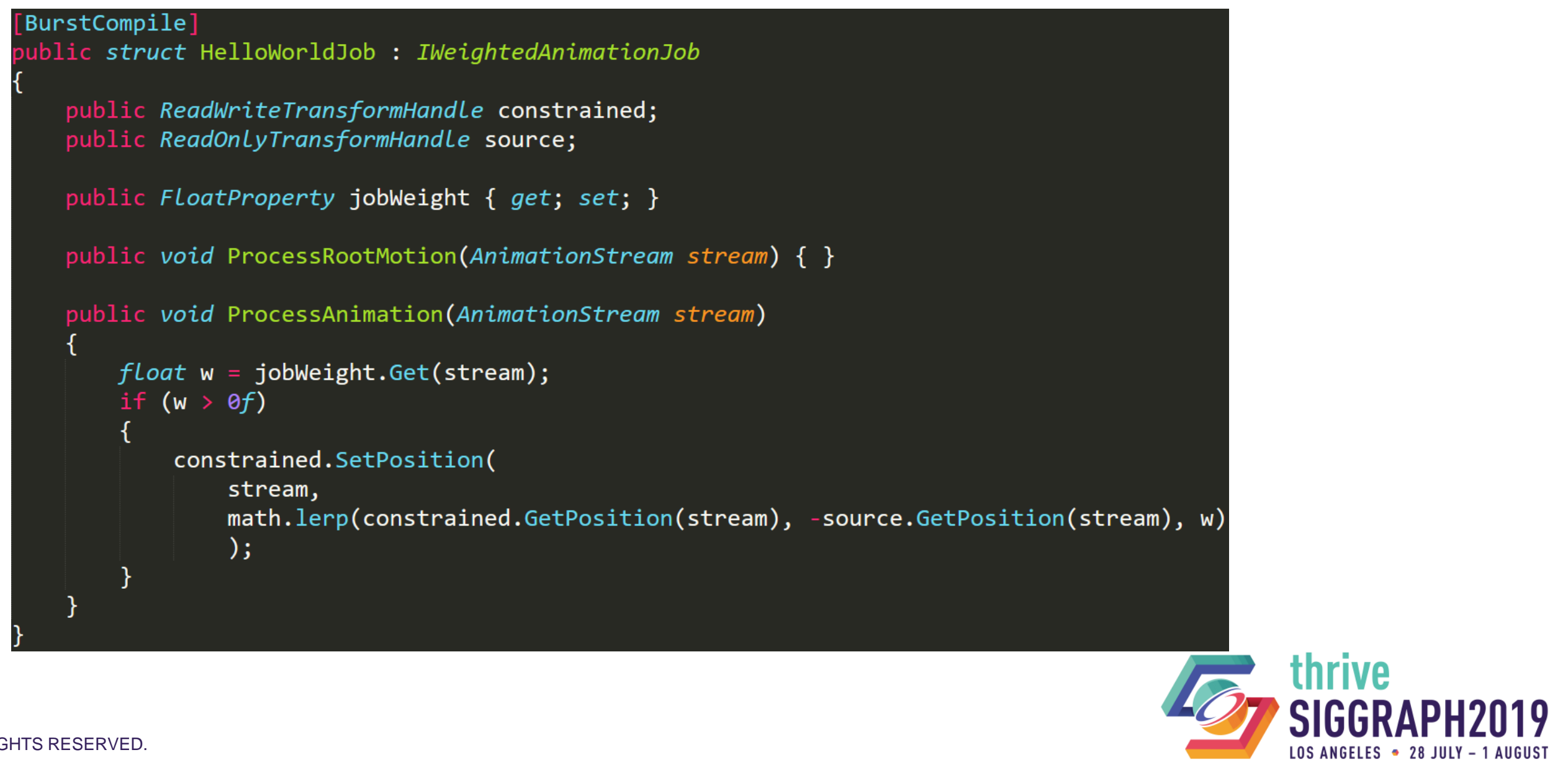




\section{SCRIPTING A CUSTOM CONSTRAINT}

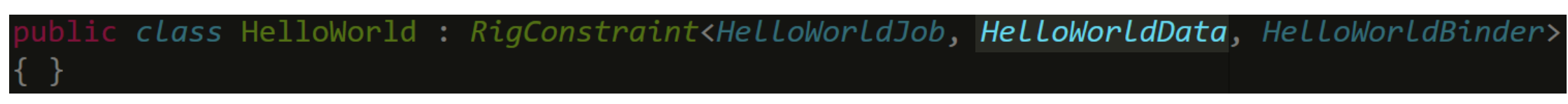

HelloWorldData is an IAnimationJobData

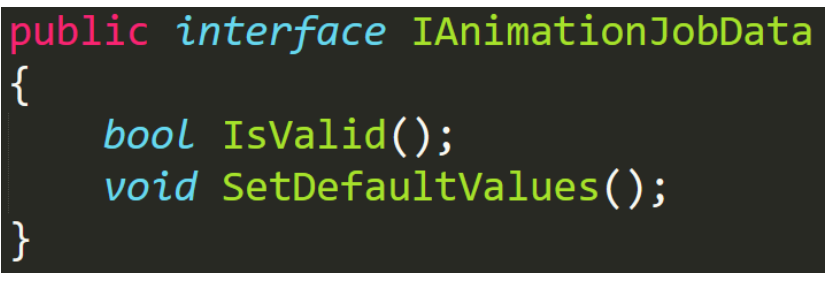

Contains necessary data to create the job 


\section{SCRIPTING A CUSTOM CONSTRAINT}

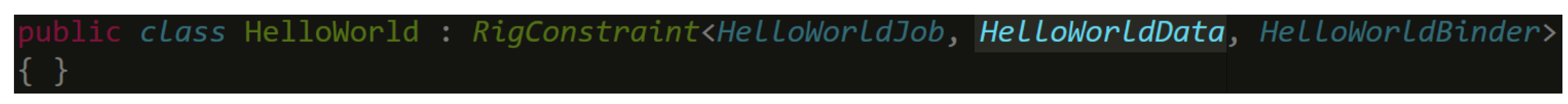

HelloWorldData is an IAnimationJobData

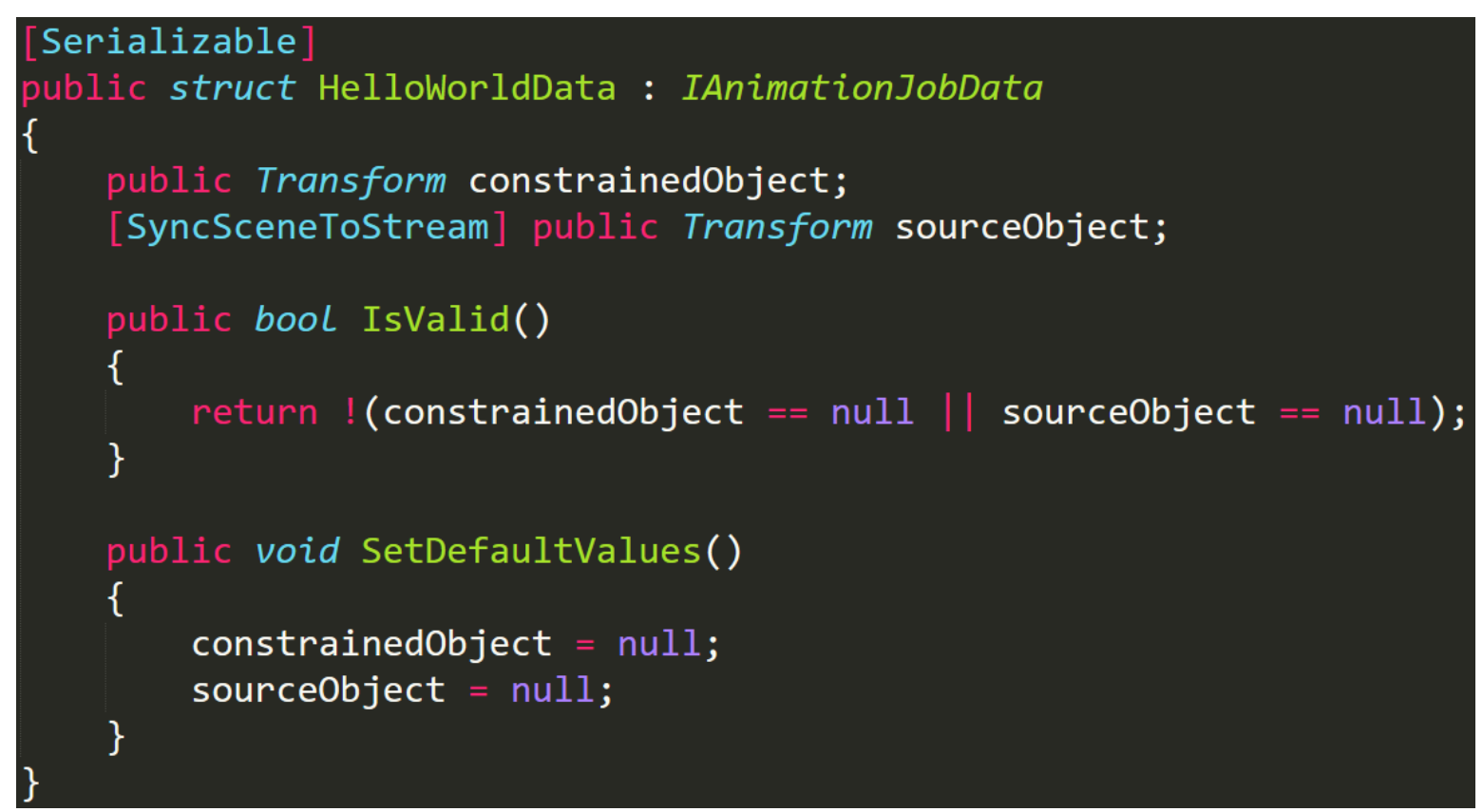




\section{SCRIPTING A CUSTOM CONSTRAINT}

\section{class Helloworld

HelloWorldBinder is an AnimationJobBinder

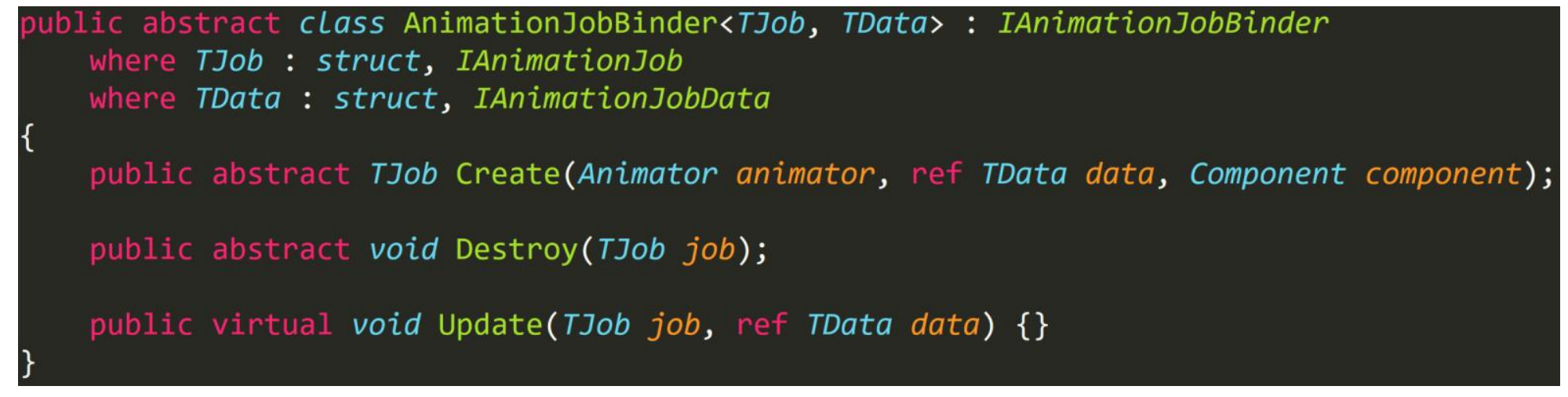

Create/Destroy/Update an IAnimationJob given some IAnimationJobData 


\section{SCRIPTING A CUSTOM CONSTRAINT}

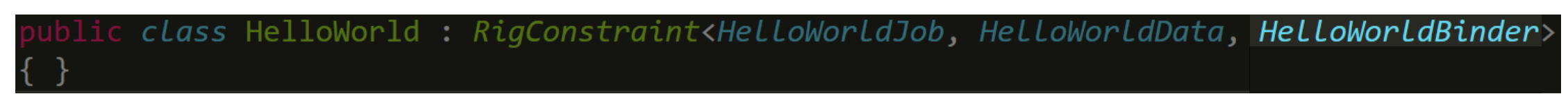

HelloWorldBinder is an AnimationJobBinder

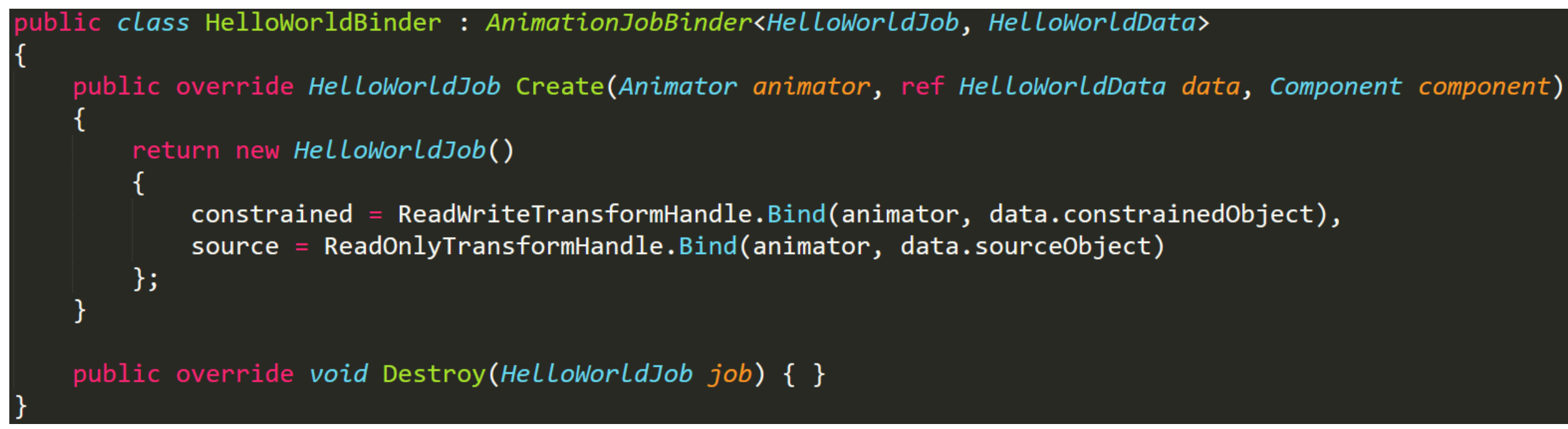




\section{SCRIPTING A CUSTOM CONSTRAINT}

\section{public class HelloWorld : RigConstraint<HelloWorldJob, HelloWorldData, HelloWorldBinder>}

\{\}
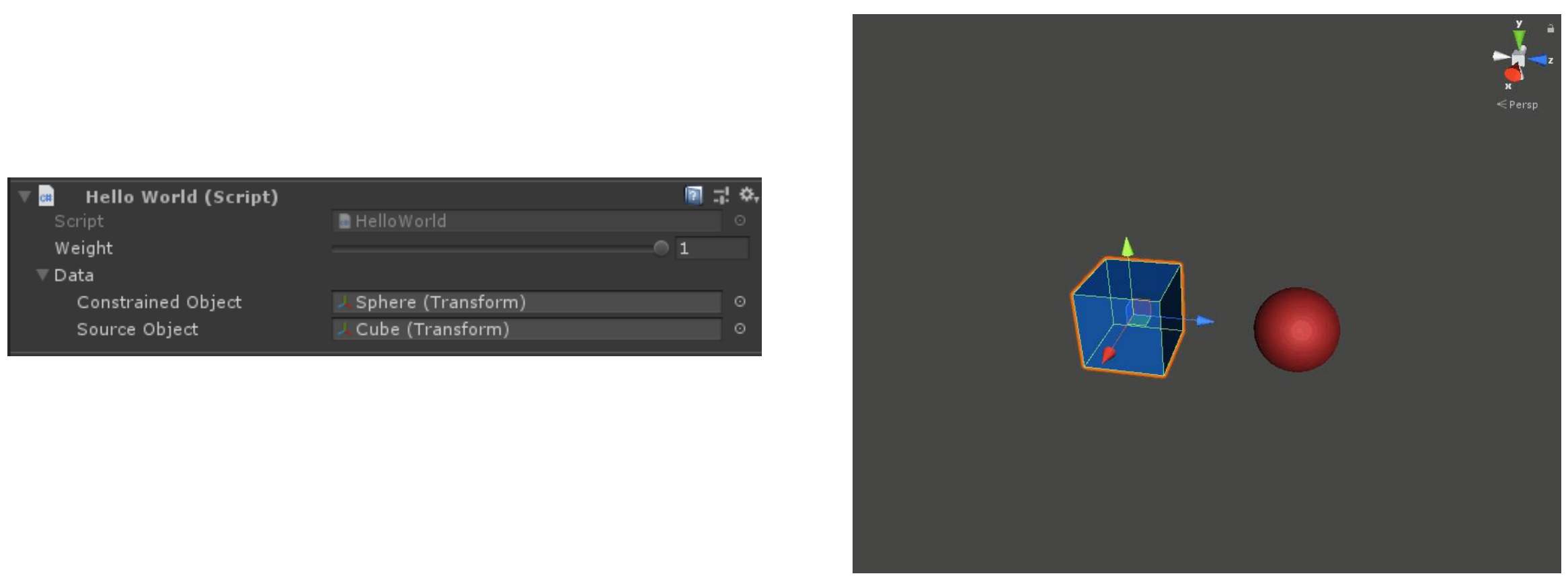


\section{LIVE DEMO}

Lets rework the HelloWord constraint

Open the 02_WorkshopCopyLocation scene

Add toggles on the constraint to invert the axis values 


\section{INSPECTING THE PLAYABLE GRAPH}

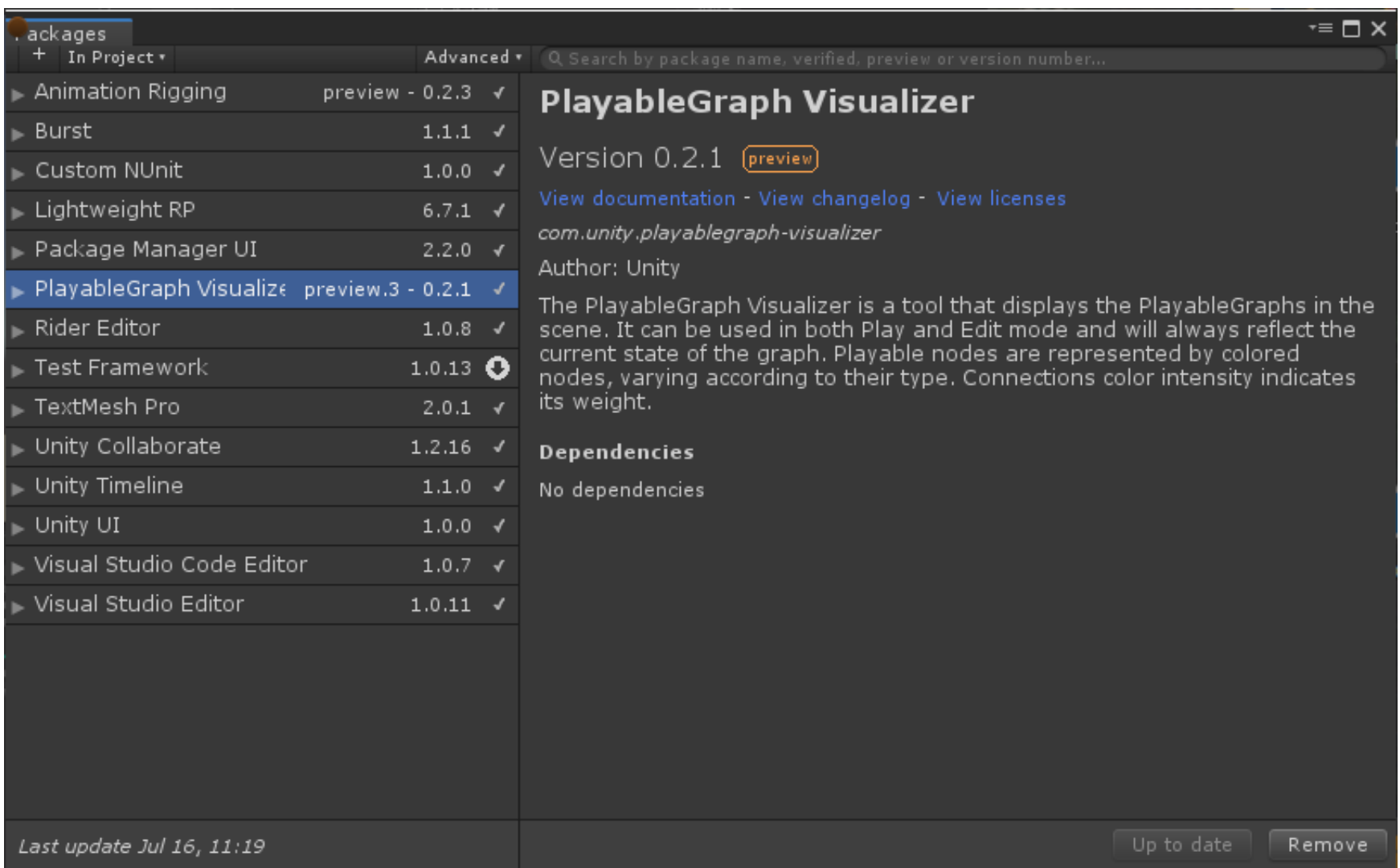

Window Help

Next Window

Previous Window

Layouts

Internal

Unity Connect

Asset Store

Package Manager

Asset Management

TextMeshPro

General

Rendering

Animation

Audio

Sequencing

Analysis

2D

Al

XR

UI

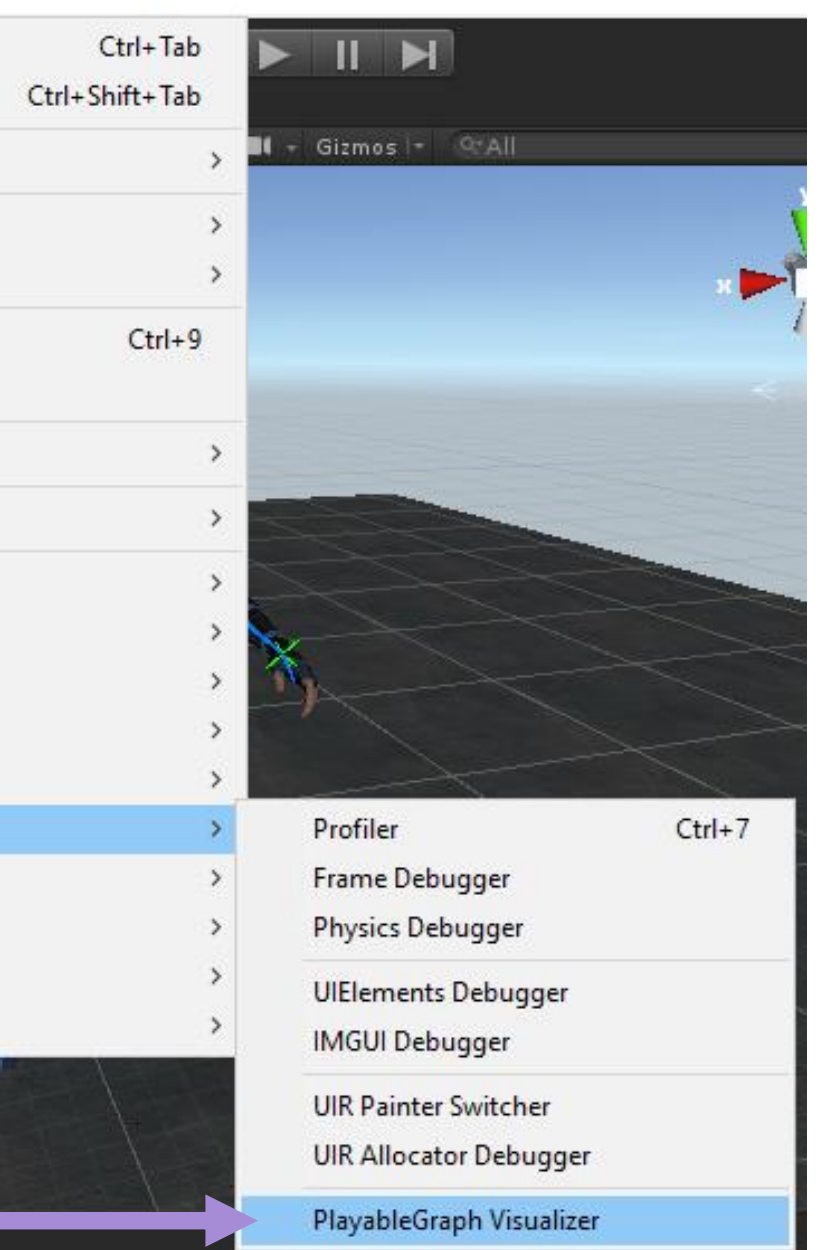

thrive

SIGGRAPH2019 


\section{INSPECTING THE PLAYABLE GRAPH}

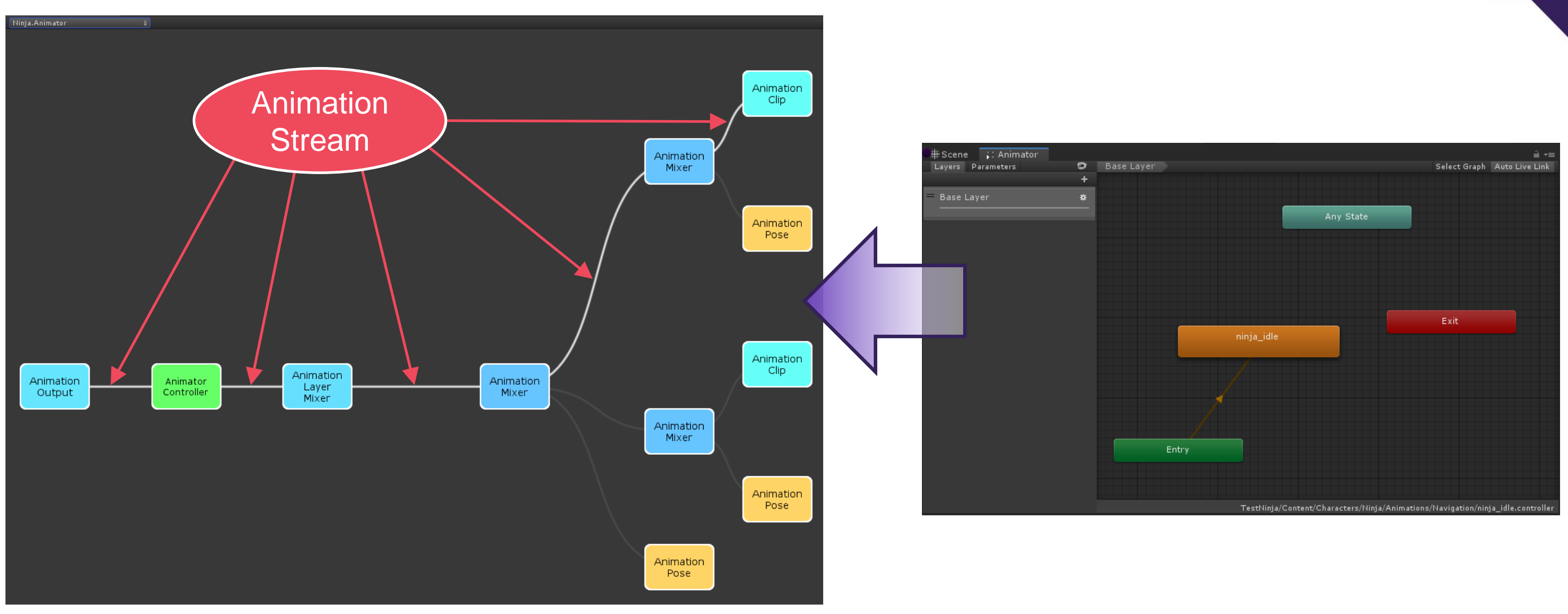




\section{INSPECTING THE PLAYABLE GRAPH}

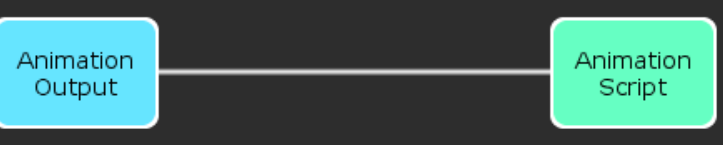




\section{INSPECTING THE PLAYABLE GRAPH}

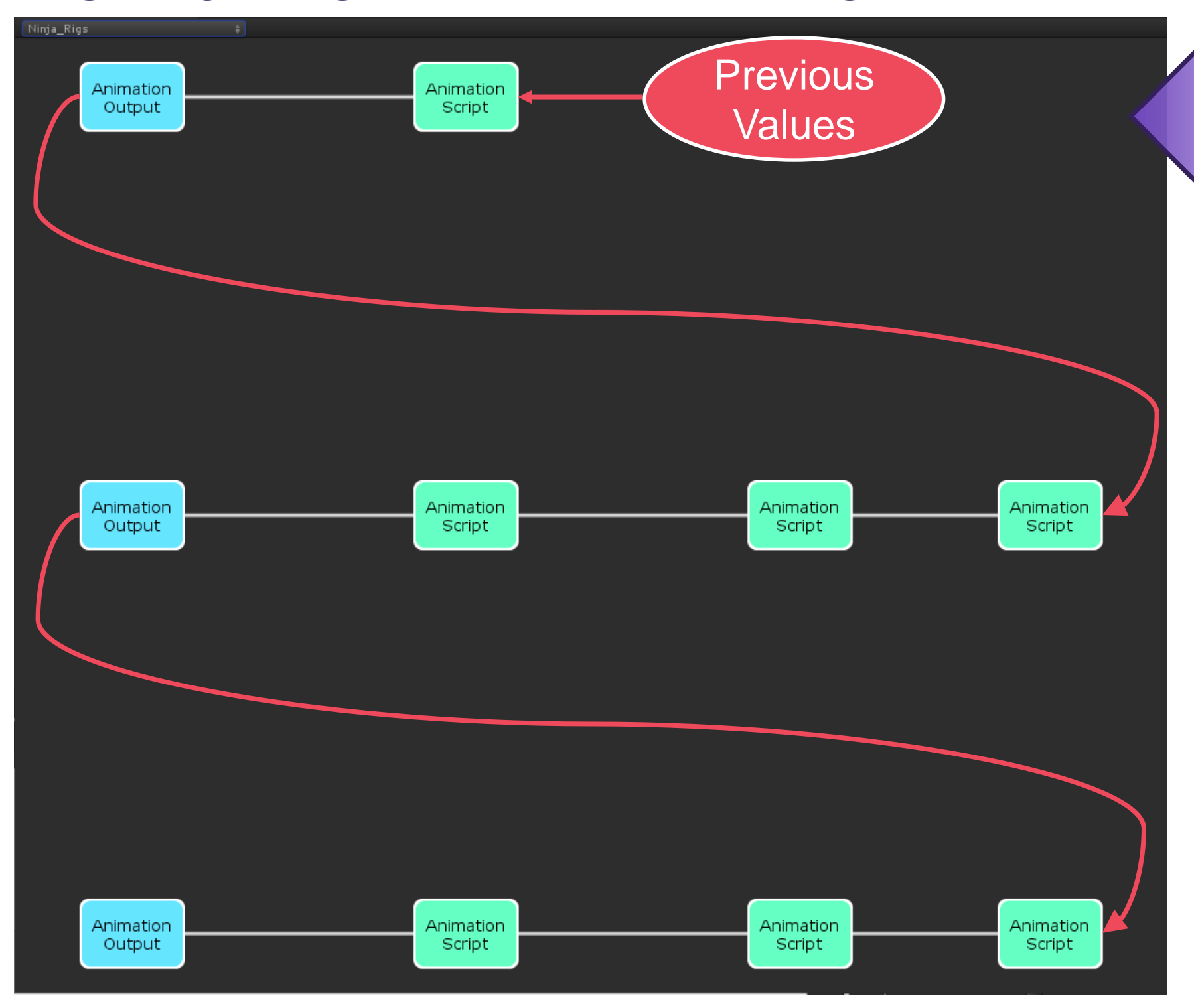




\section{INSPECTING THE PLAYABLE GRAPH}

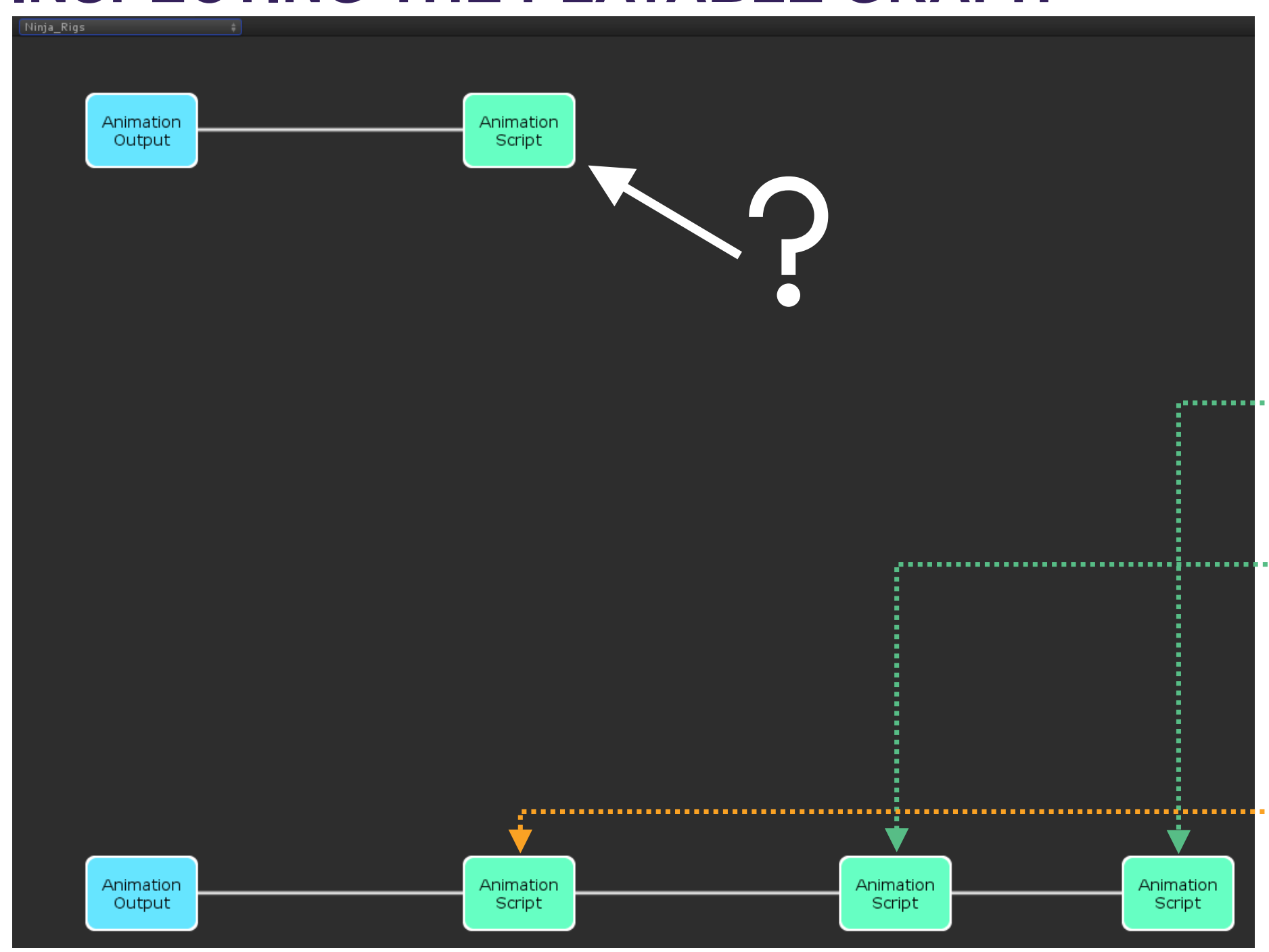

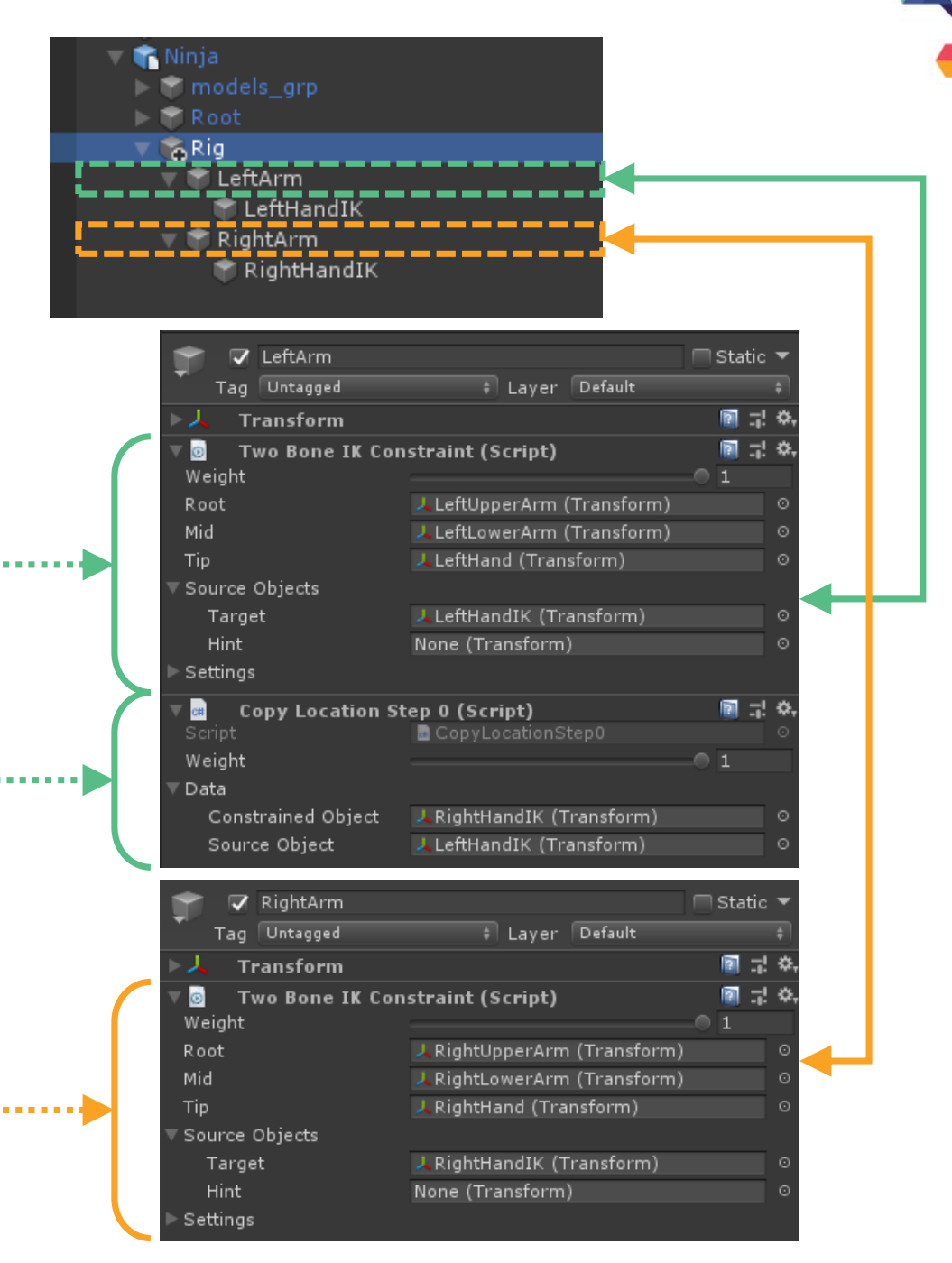

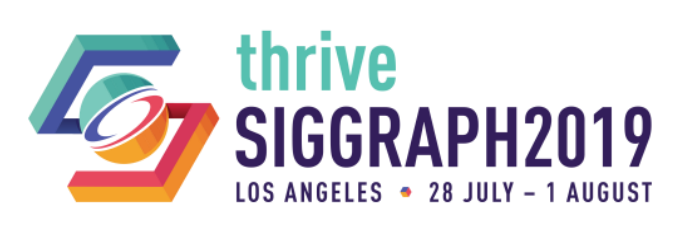




\section{SYNC VALUES TO ANIMATION STREAM}

- First job to run before evaluating any of the rig layers

- Pushes latest scene values to the animation stream

- Only if these have NOT previously been animated

- Makes latest values available to all downstream jobs

- Flag constraint fields using [SyncSceneToStream]

- Works on a limited set of data types:

- Float, Int, Bool, Vector[2,3,4], Quaternions, Vector3Int, Vector3Bool

- Transform, Transform[], WeightedTransform, WeightedTransformArray 


\section{SYNC VALUES TO ANIMATION STREAM}

- RigTransform component

- A quick way to sync transforms that are not referenced by any constraints

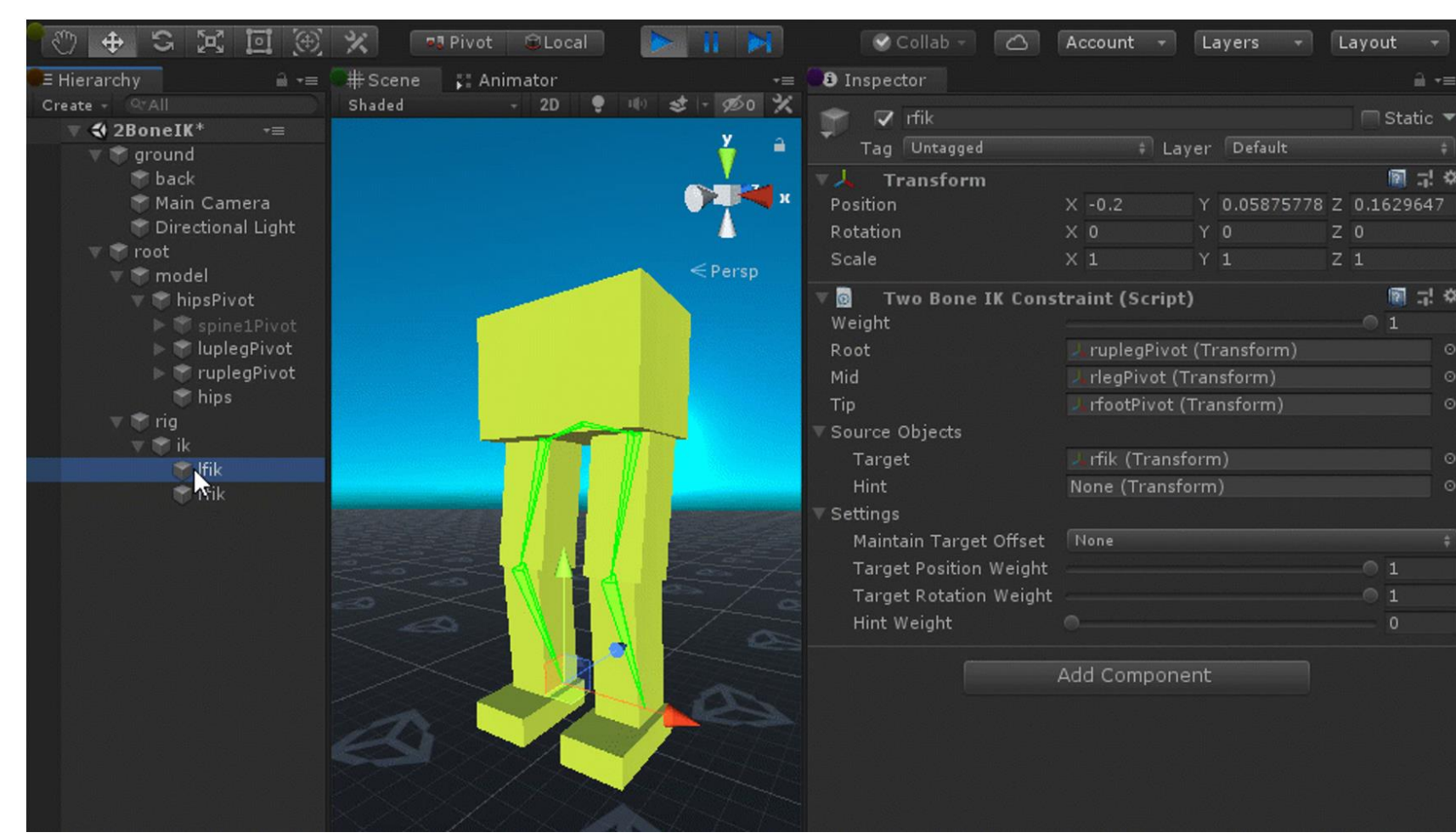




\section{LIVE DEMO}

- Let's rework our CopyLocation constraint to make our newly added axis toggles dynamic and part of the AnimationStream. 


\section{BUILDING A TWIST CHAIN CONSTRAINT}

- We're proposing a bidirectional twist chain constraint driven by two effectors.

We'll be limiting this constraint to animating rotation only.
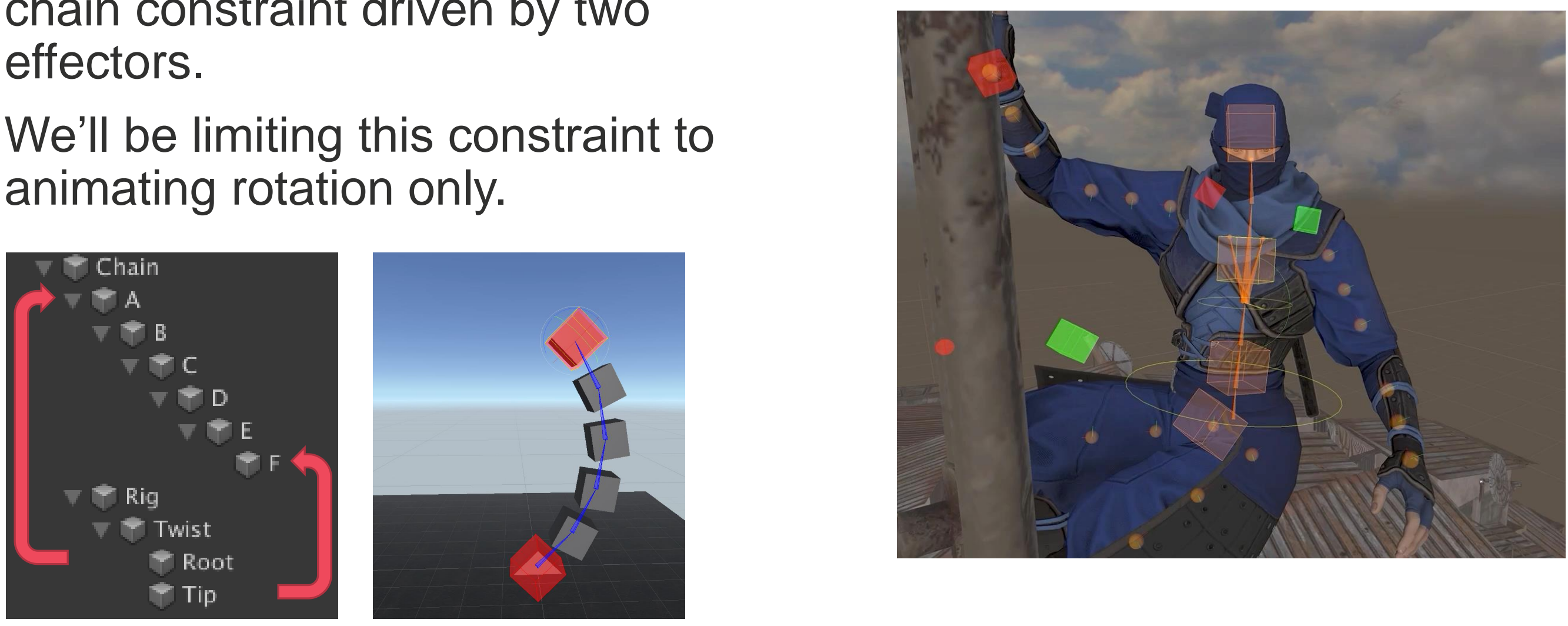


\section{BUILDING A TWIST CHAIN CONSTRAINT}

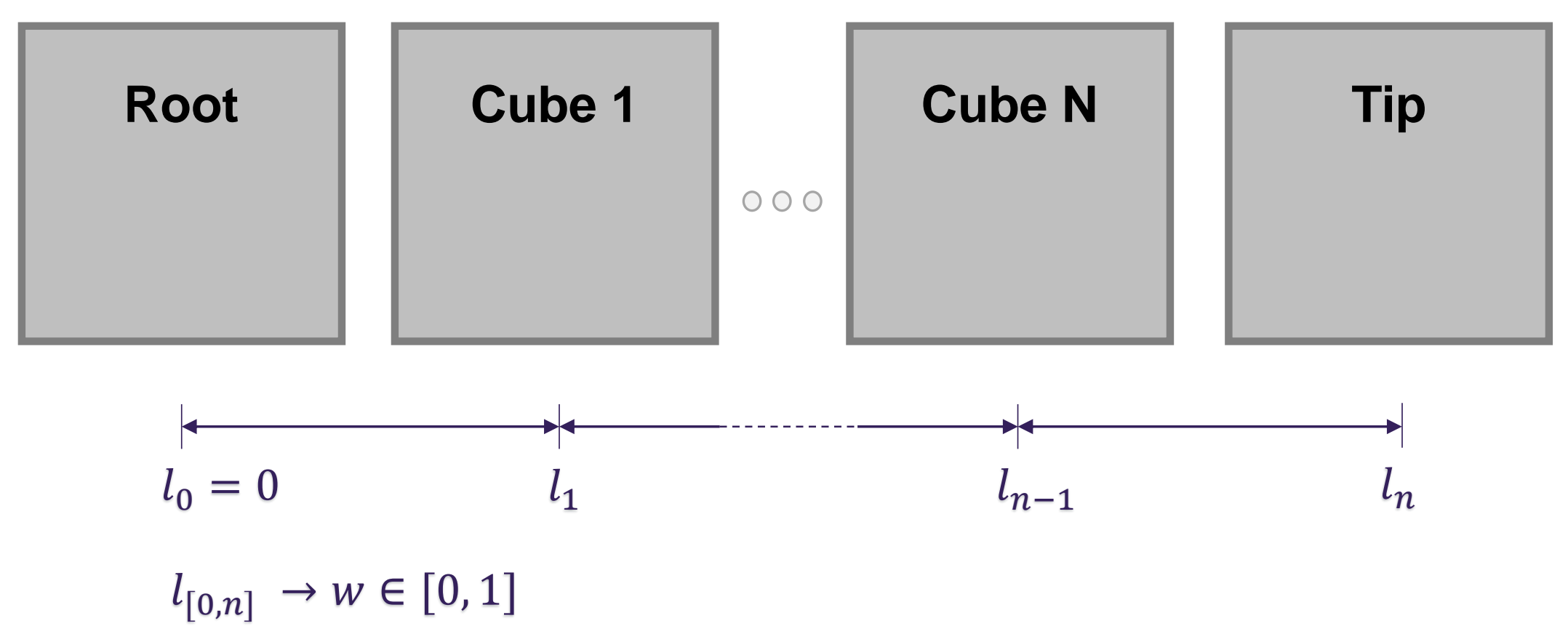




\section{BUILDING A TWIST CHAIN CONSTRAINT}

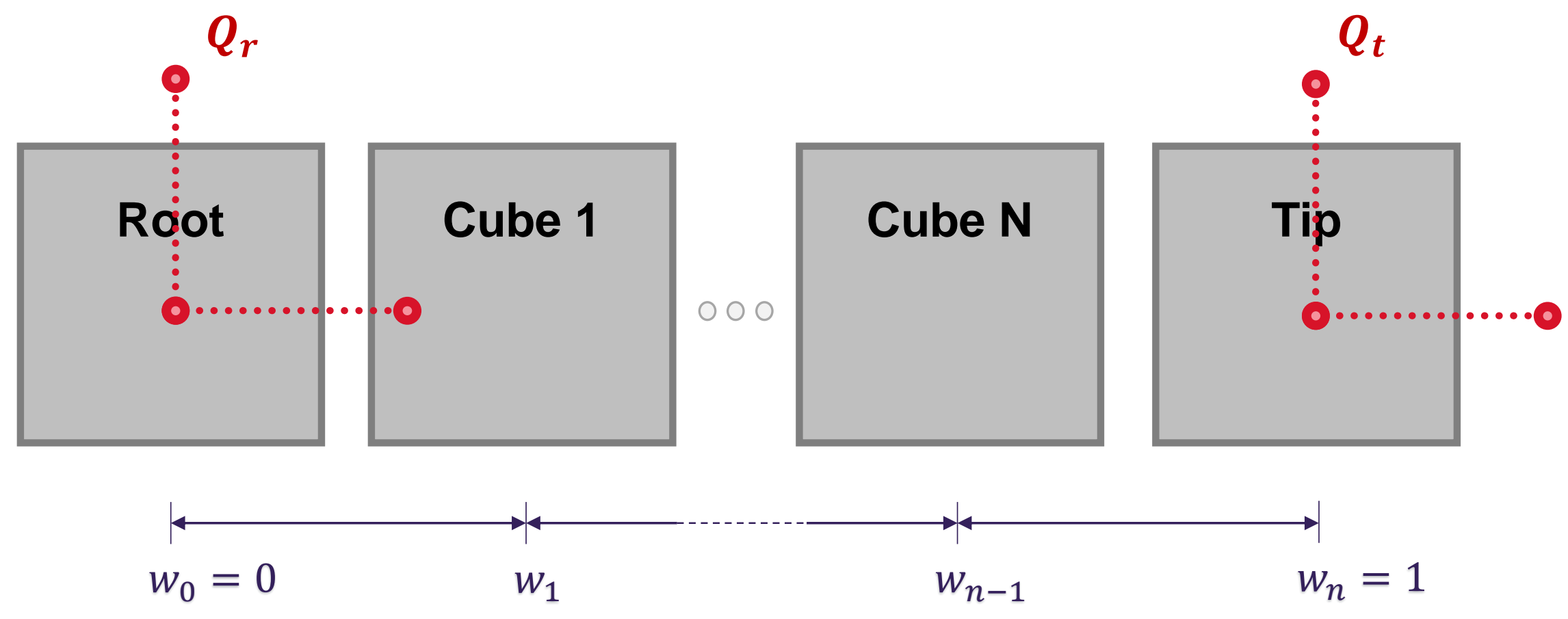




\section{BUILDING A TWIST CHAIN CONSTRAINT}

$$
w \in[\mathbf{0}, \mathbf{1}]
$$

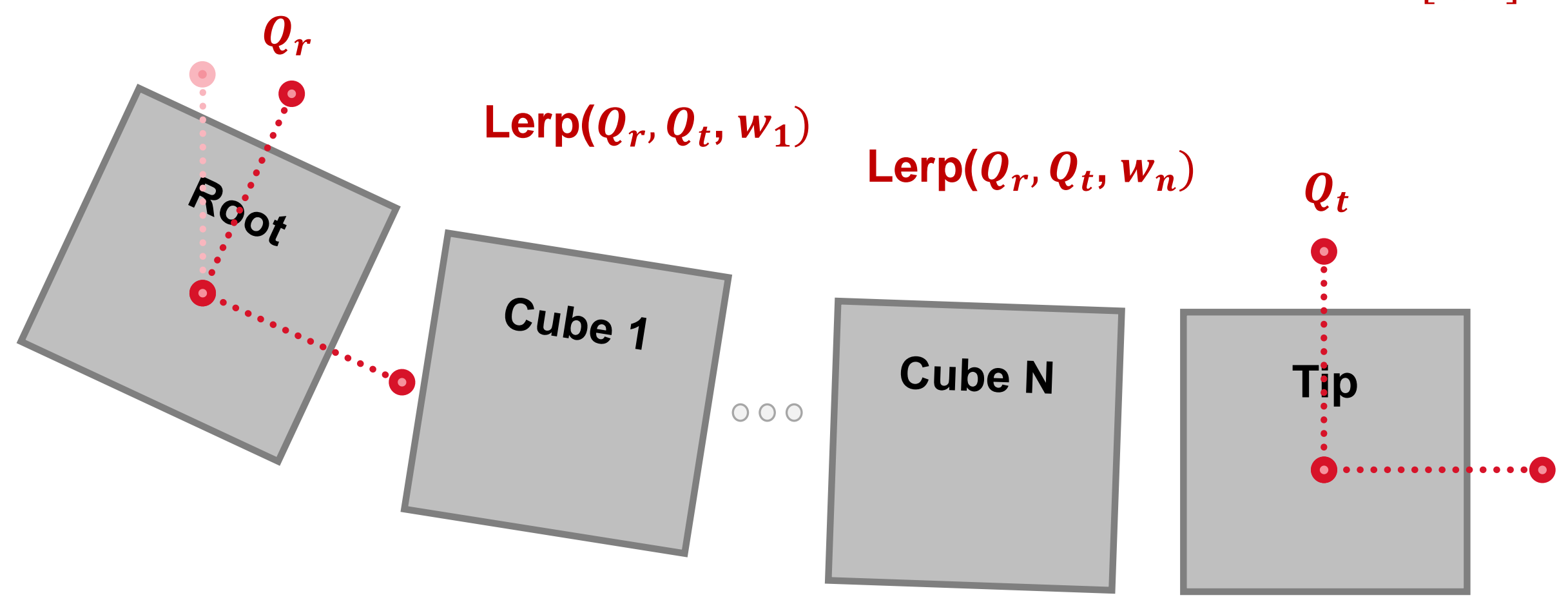




\section{BUILDING A TWIST CHAIN CONSTRAINT}

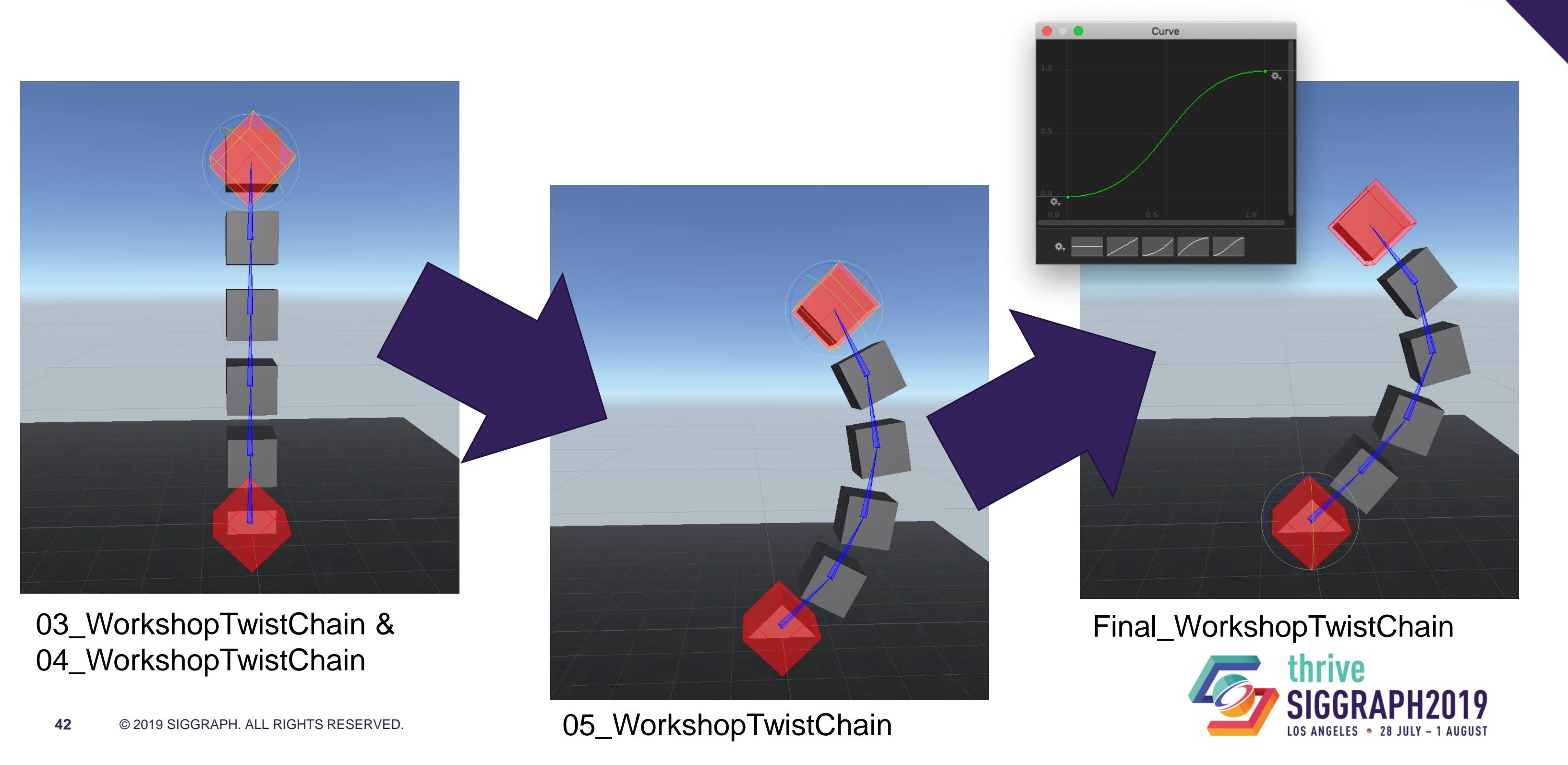




\section{BUILDING A TWIST CHAIN CONSTRAINT}

- Open scene 03_WorkshopTwistChain.

- The scene is already setup with a basic hierarchy and RigBuilder.

- The constraint TwistChainStep0 will execute, but does nothing at the moment.

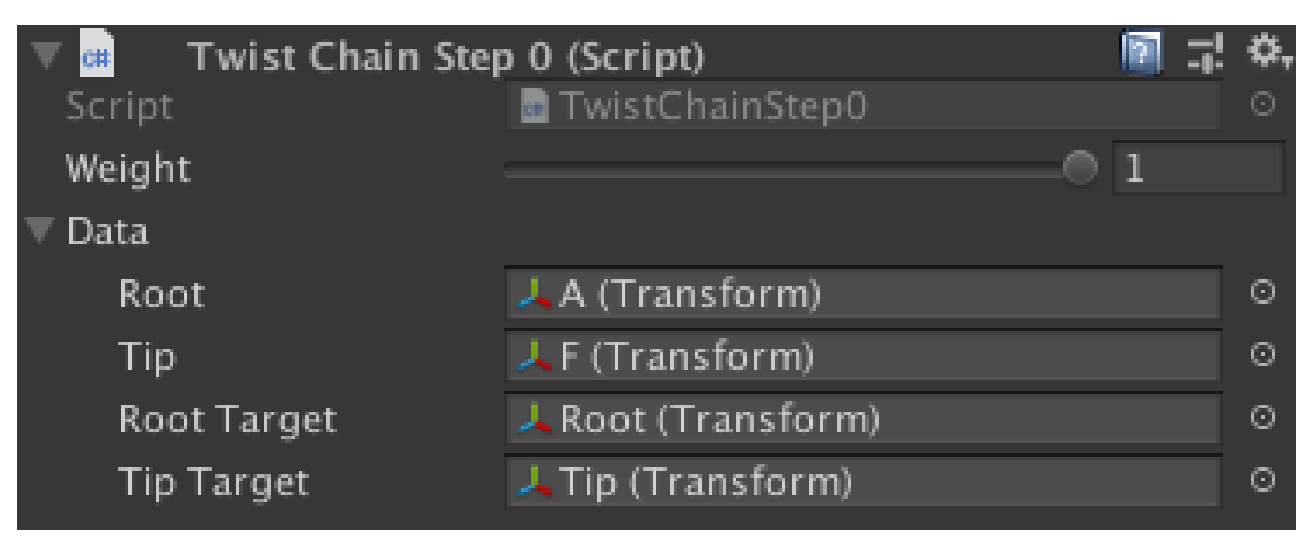

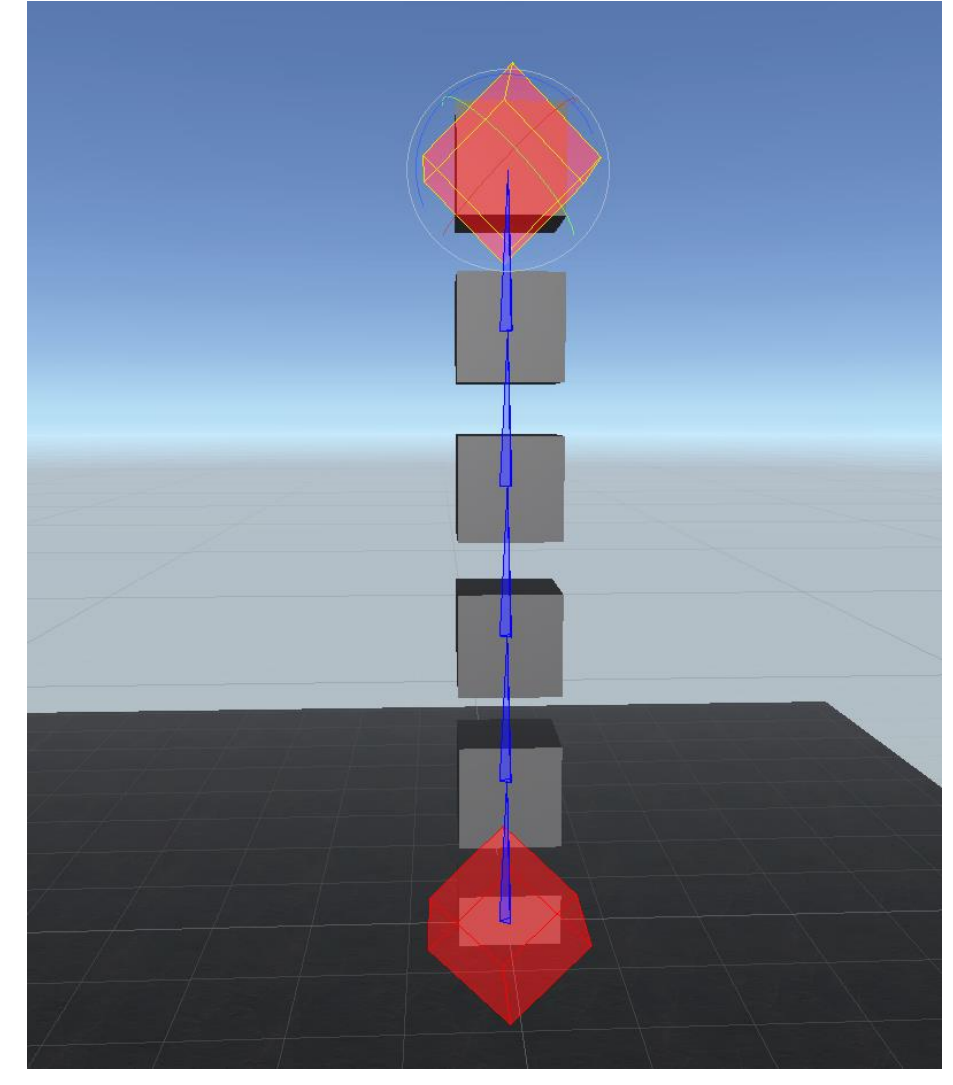

TwistChainStep0.unity 


\section{BUILDING A TWIST CHAIN CONSTRAINT}

\section{- TwistChainData}

\section{[System.Serializable]}

oublic struct TwistChainstep@Data : IAnimationJobData

\section{public Transform root;}

public Transform tip;

[SyncSceneToStream] public Transform rootTarget;

[SyncSceneToStream] public Transform tipTarget;

bool IAnimationJobData.IsValid() $\Rightarrow$ ! (root == null || tip $==$ null || ! tip.IsChildof(root) || rootTarget $==$ null || tipTarget $==$ null);

void IAnimationJobData.SetDefaultValues()

\{

root $=$ tip $=$ rootTarget $=$ tipTarget $=$ null $;$ 


\section{BUILDING A TWIST CHAIN CONSTRAINT}

TwistChainData

[SyncSceneToStream] public Transform rootTarget;

[SyncSceneToStream] public Transform tipTarget;

IAnimationJobData.IsValid () $\Rightarrow !$ ( root

null || tip

null

tip.IsChild0f (root)

rootTarget

null

tipTarget

IAnimationJobData. SetDefaultValues()

root $=$ tip $=$ rootTarget $=$ tipTarget $=$ null $;$ 


\section{BUILDING A TWIST CHAIN CONSTRAINT}

- TwistChainData
_SyncSceneToStream] public Transform rootTarget;
[SyncSceneToStream] public Transform tipTarget;

IAnimationJobData.Isvalid () $\Rightarrow !($ root

tip

nult

tip. IsChildof (root)

rootTarget

nutl

tipTarget

IAnimationJobData . SetDefaultValues ()

root $=$ tip $=$ rootTarget $=$ tipTarget $=$ null $;$ 


\section{BUILDING A TWIST CHAIN CONSTRAINT}

- TwistChainData

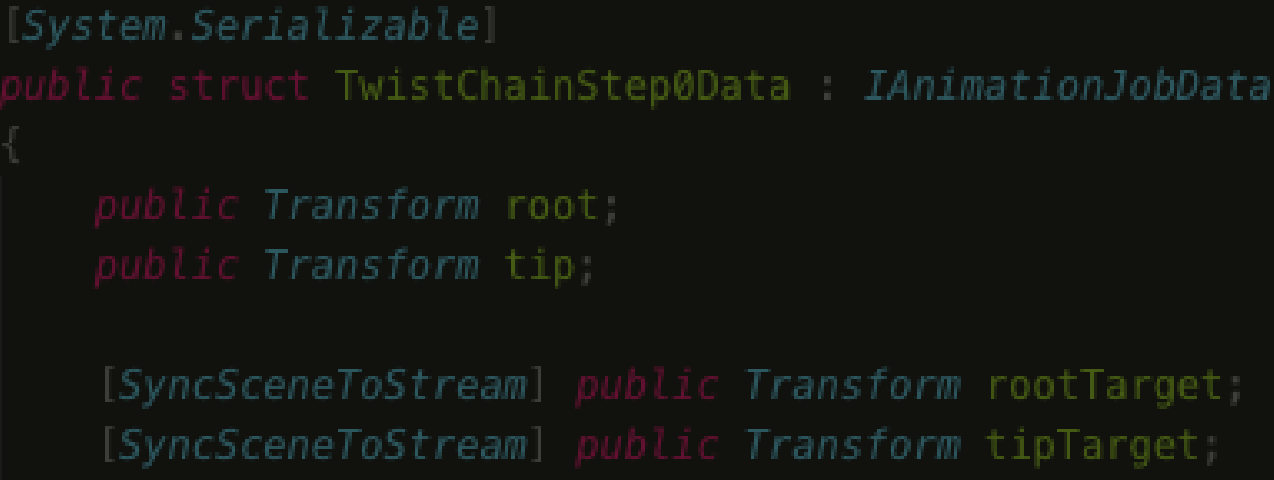




\section{BUILDING A TWIST CHAIN CONSTRAINT}

TwistChainData

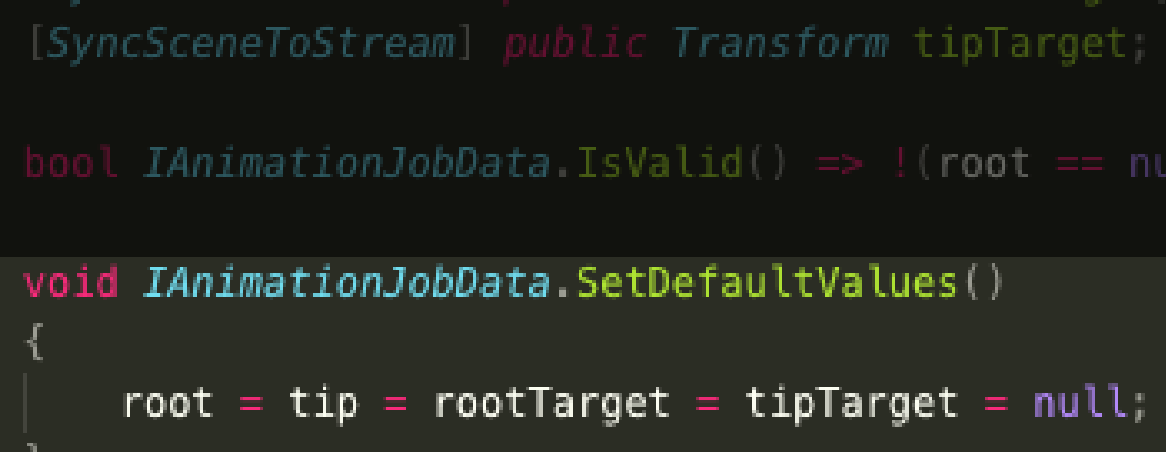




\section{BUILDING A TWIST CHAIN CONSTRAINT}

\section{TwistChainJob}

[Unity.Burst.BurstCompile]

public struct TwistChainStep0Job : IWeightedAnimationJob

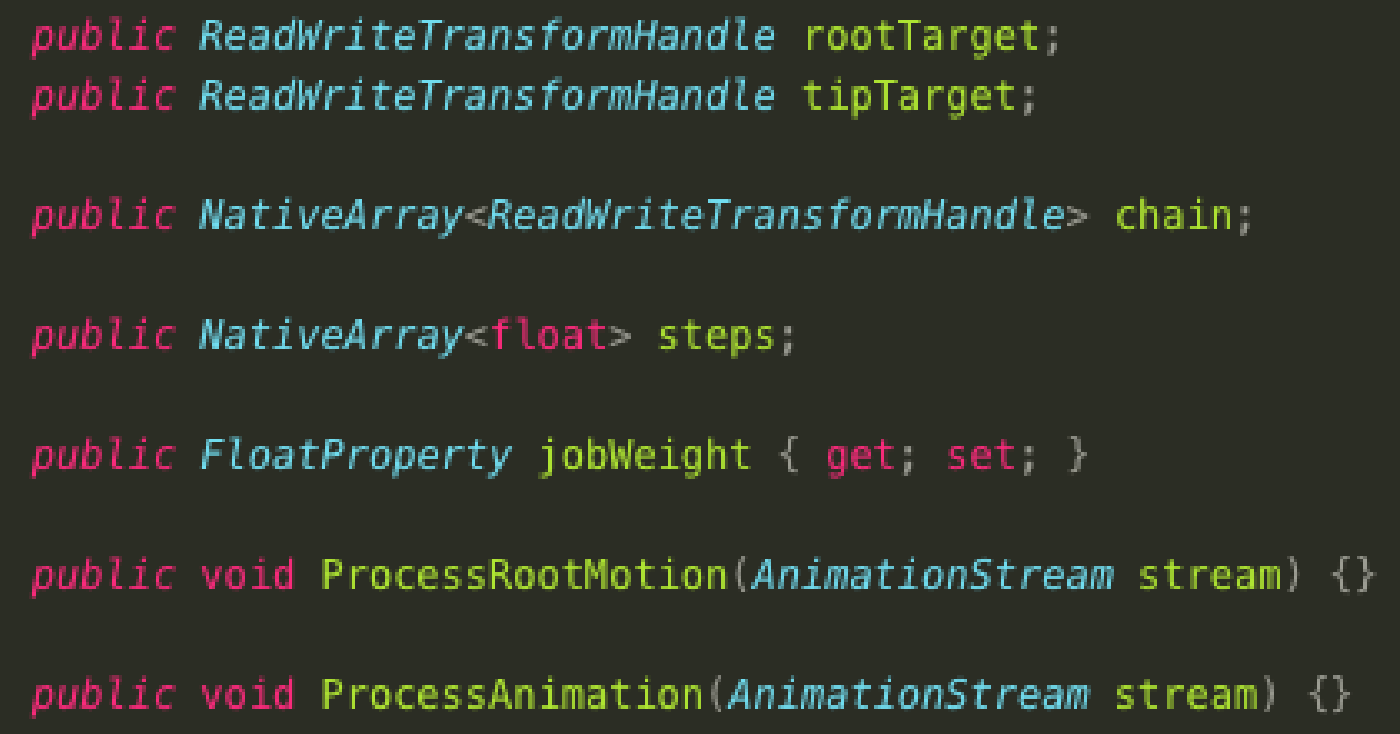




\section{BUILDING A TWIST CHAIN CONSTRAINT}

\section{- TwistChainJob}

[Unity.Burst.BurstCompile]
oublic struct TwistChainStepo]ob : IWeightedAnimationJob

public ReadWriteTransformHandle rootTarget;

public ReadWriteTransformHandle tipTarget;

NativeArray<ReadWriteTransformHandle> chain;

NativeArray<float > steps;

FloatProperty jobleight \{ get; set; \}

ProcessRootMotion(AnimationStream stream) \{\} 


\section{BUILDING A TWIST CHAIN CONSTRAINT}

\section{- TwistChainJob}

[Unity.Burst.BurstCompile]
public struct TwistChainstepo]ob : IWeightedAnimationJob

\section{ReadWriteTransformHandle rootTarget;}

ReadWriteTransformHandle tipTarget;

public NativeArray<ReadWriteTransformHandle> chain;

public NativeArray<float> steps;

FloatProperty jobleight \{ get; set; \} 


\section{BUILDING A TWIST CHAIN CONSTRAINT}

\section{- TwistChainJob}

[Unity.Burst.BurstCompile]
public struct TwistChainstepo]ob : IWeightedAnimationJob

ReadWriteTransformHandle rootTarget;

ReadWriteTransformHandle tipTarget;

NativeArray<ReadWriteTransformHandle $>$ chain;

NativeArray<float> steps;

public FloatProperty jobWeight \{ get; set; \} 


\section{BUILDING A TWIST CHAIN CONSTRAINT}

- TwistChainJob

[Unity.Burst.BurstCompile]
Dublic struct TwistChainStep0]ob : IWeightedAnimationJob
public ReadWriteTransformHandle rootTarget;
public ReadWriteTransformHandle tipTarget;
public NativeArray<ReadWriteTransformHandle> chain;
public NativeArray<float> steps;
public FloatProperty joblleight \{ get; set; \} 


\section{BUILDING A TWIST CHAIN CONSTRAINT}

\section{- TwistChainJobBinder}

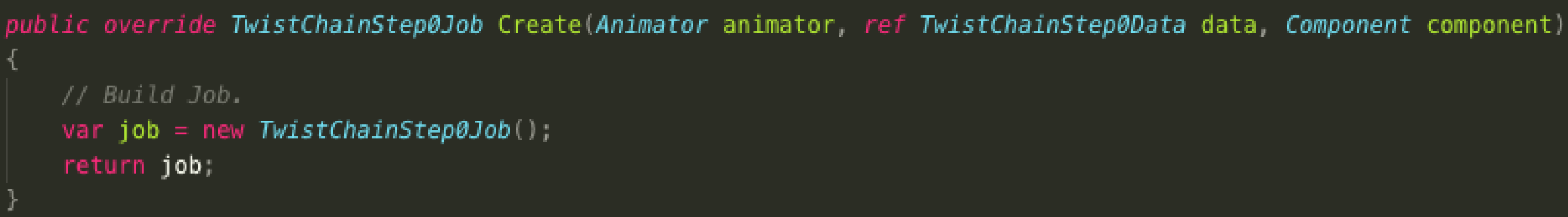




\section{BUILDING A TWIST CHAIN CONSTRAINT}

Finishing up TwistChainJob

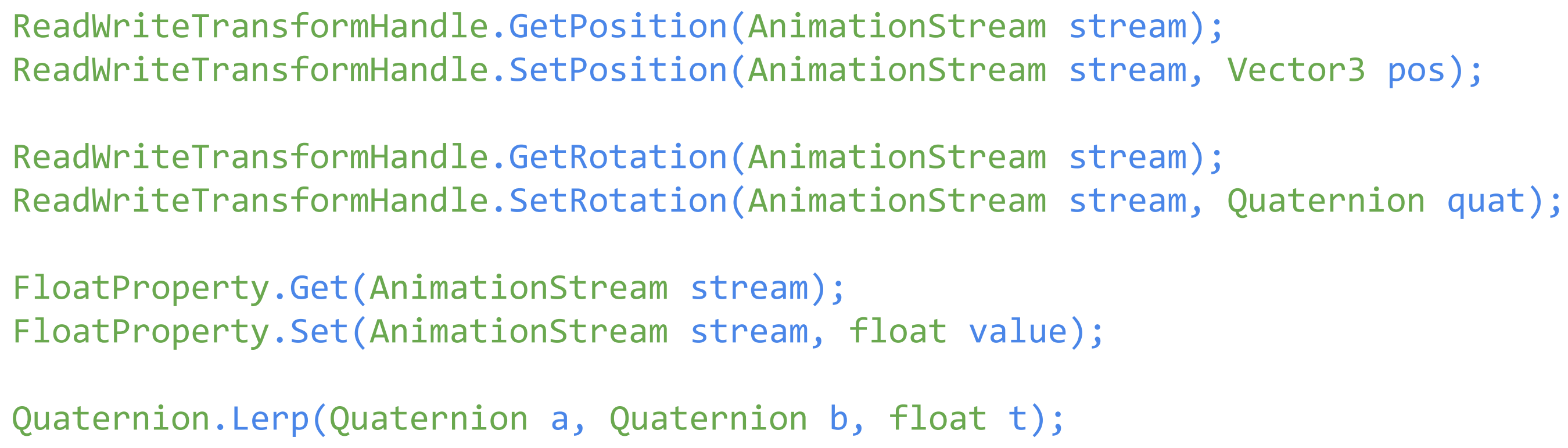




\section{BUILDING A TWIST CHAIN CONSTRAINT}

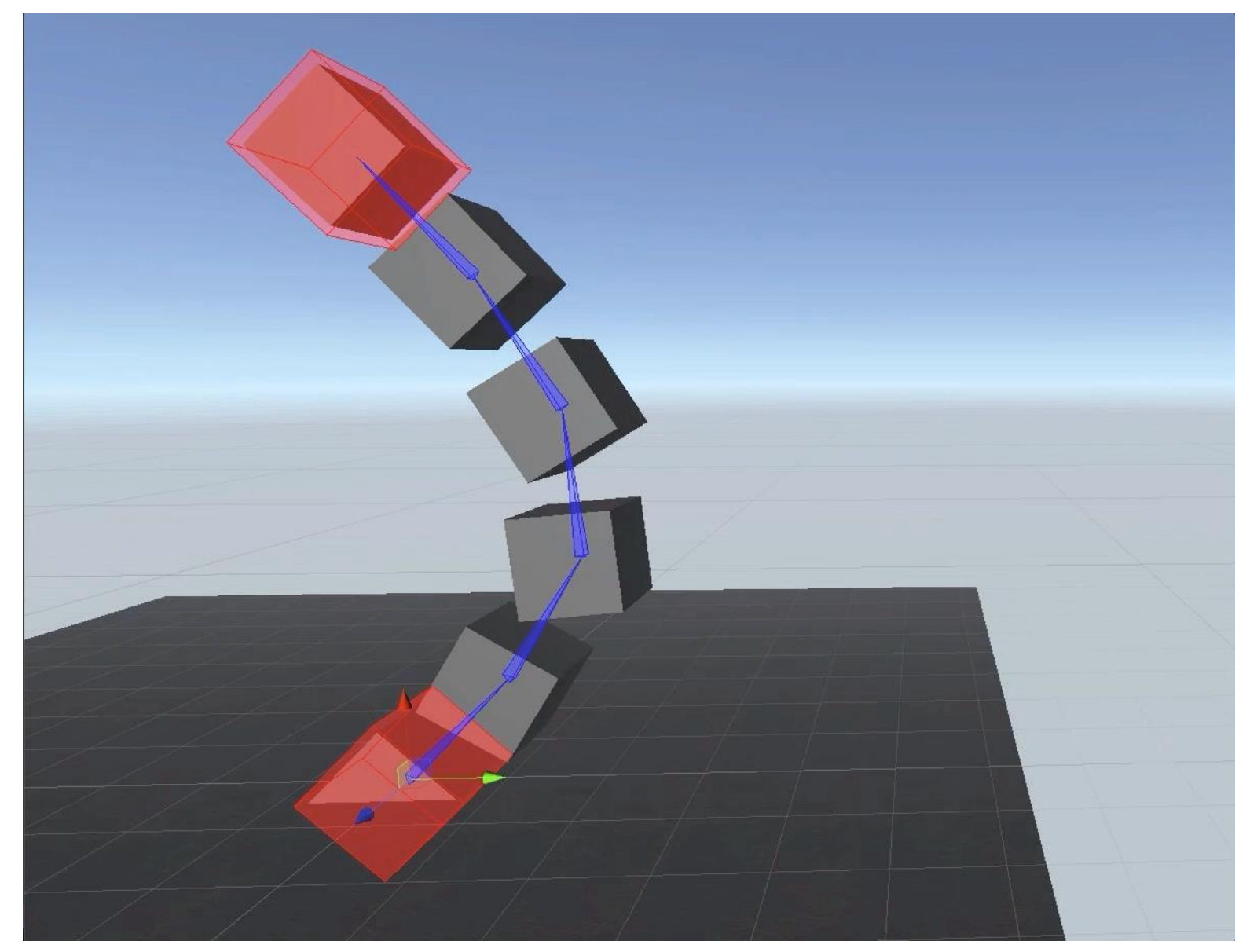




\section{IMPROVING THE TWIST CHAIN CONSTRAINT}

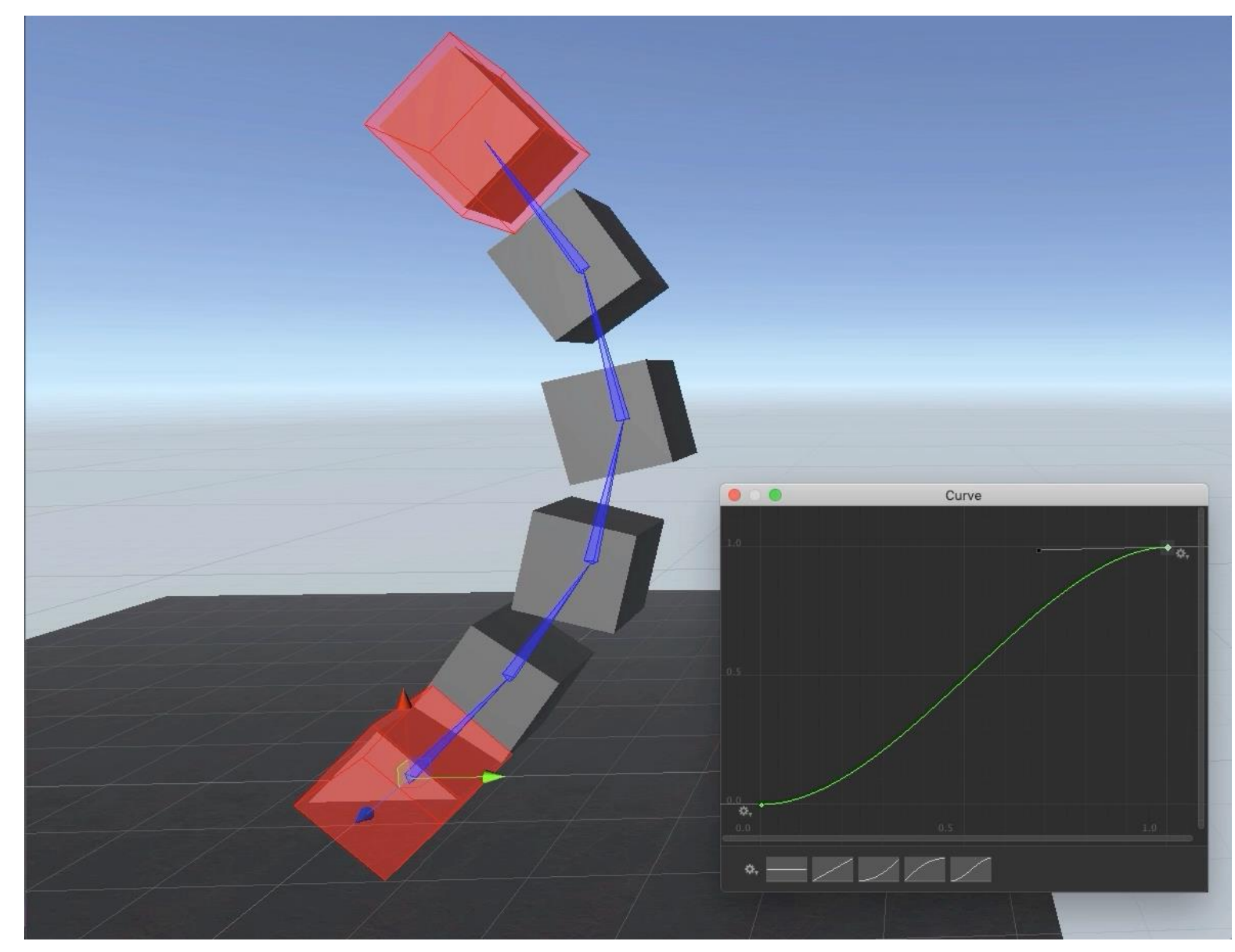




\section{IMPROVING THE TWIST CHAIN CONSTRAINT}
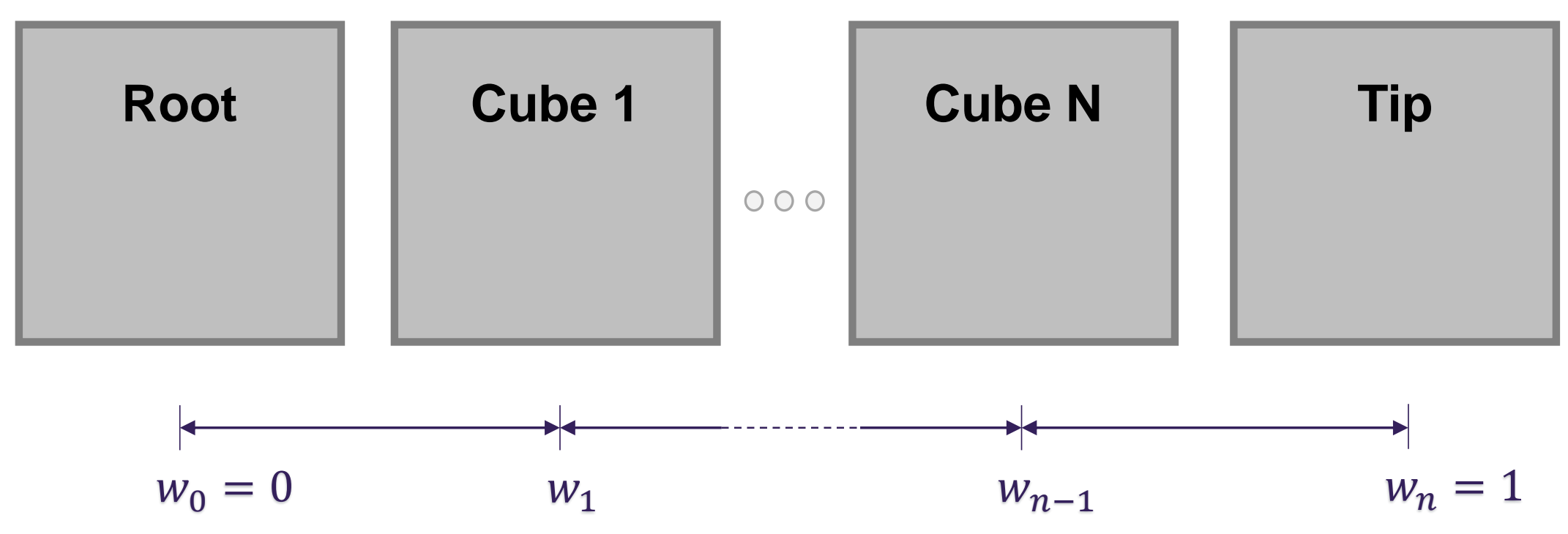


\section{IMPROVING THE TWIST CHAIN CONSTRAINT}

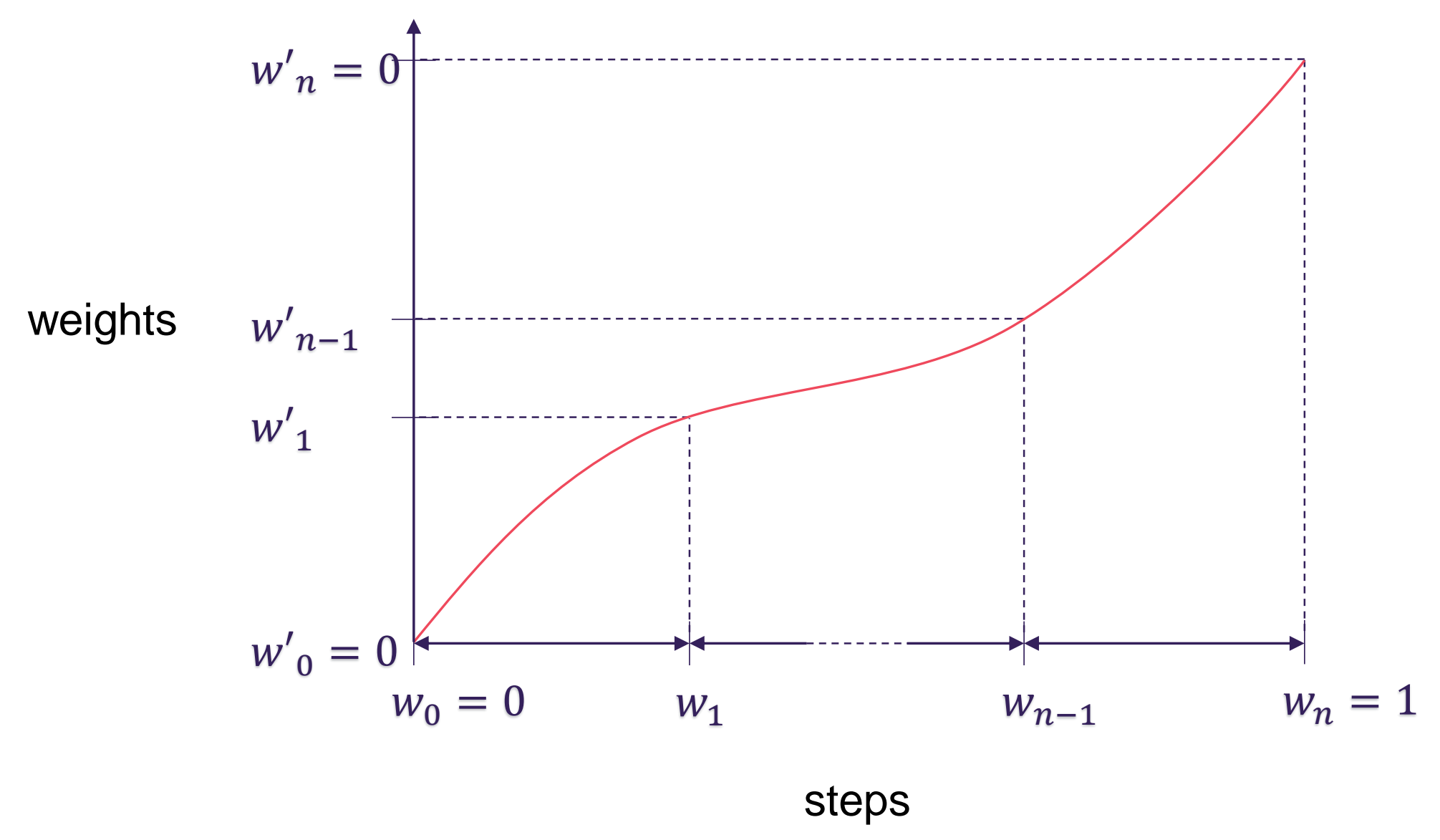




\section{IMPROVING THE TWIST CHAIN CONSTRAINT}

- Customizing further...

- Animate weight parameters for more dynamic results.

- Additional twist effectors for more control.
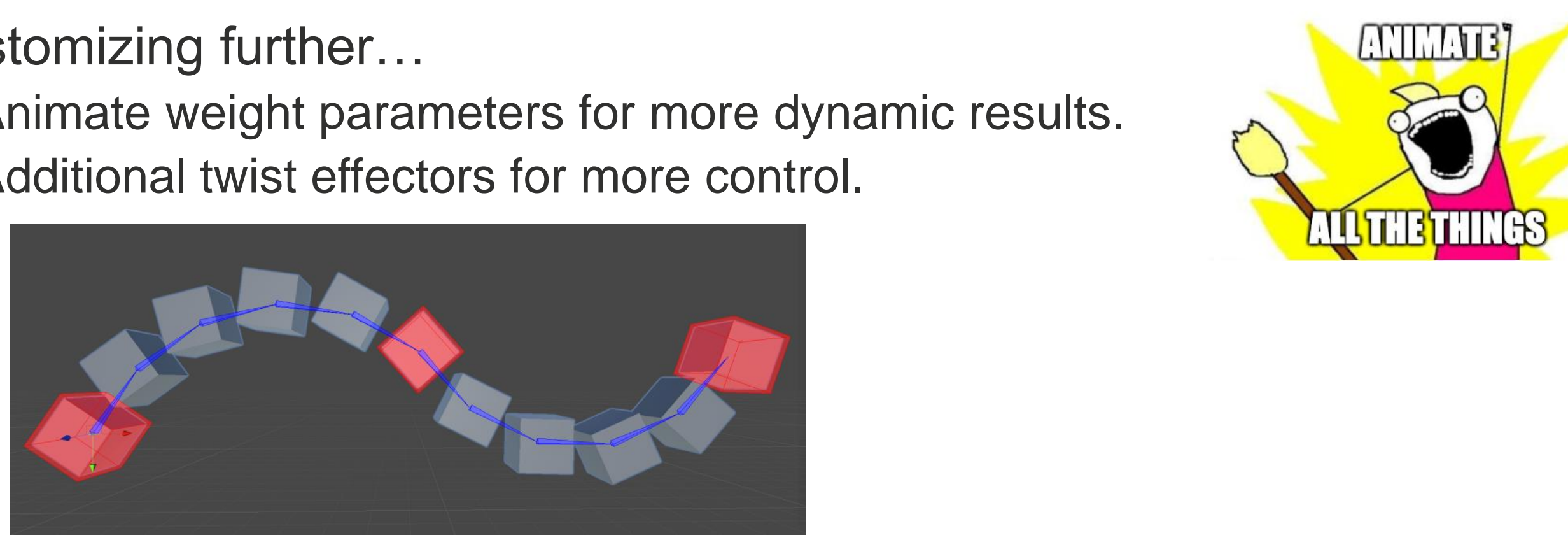

- Euler twist effectors for spins above 180 degrees.

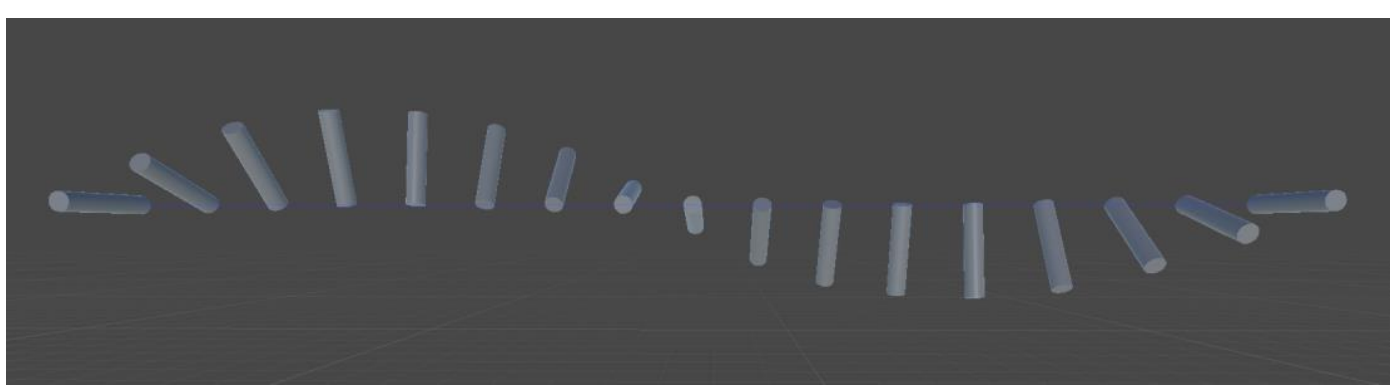


thrive

SIGGRAPH2019

LOS ANGELES - 28 JULY - 1 AUGUSt

\section{WRAPPING UP}

Setting up the spine region
- Open the scene: 06_WorkshopSpineRig

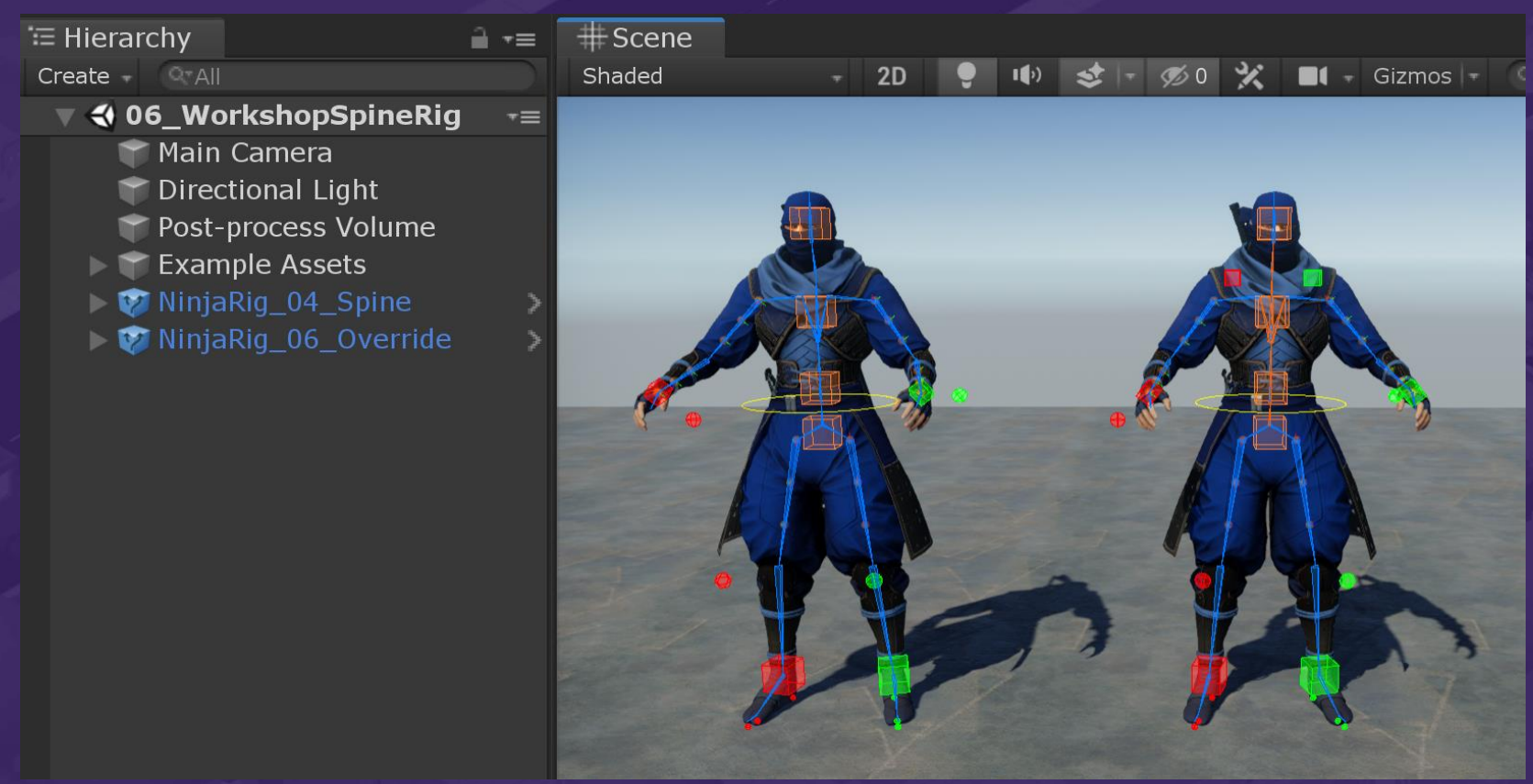




\section{SETTING UP THE SPINE REGION - COG AND PELVIS}

Spine region - Setup instructions

1. Add a new GameObject for the Spine region (child of ControlRig)

- Center of gravity - Setup instructions

1. Add a child GameObject named CenterOfGravity (child of Spine)

2. Add a Rig Transform component (so that it acts as the parent)

\section{- Pelvis - Setup instructions}

1. Add a new GameObject named Pelvis (child of CenterOfGravity)

2. Add a child GameObject named Pelvis_parentConstraint

1. Add a Multi-Parent Constraint

1. Constrained Object: Hips bone, Source Object: Pelvis_parentConstraint

2. In Settings, set Maintain Offset to None

2. Add a Multi-Referential Constraint and assign itself as the first Reference Object

3. Add a new GameObject named Pelvis_target (child of Pelvis)

1. Align this to the first Spine bone using: Animation Rigging > Align Transform

2. Customize the Effector display options

3. Assign this as a Reference Object of the Multi-Referential Constraint and set it to be the Driving object
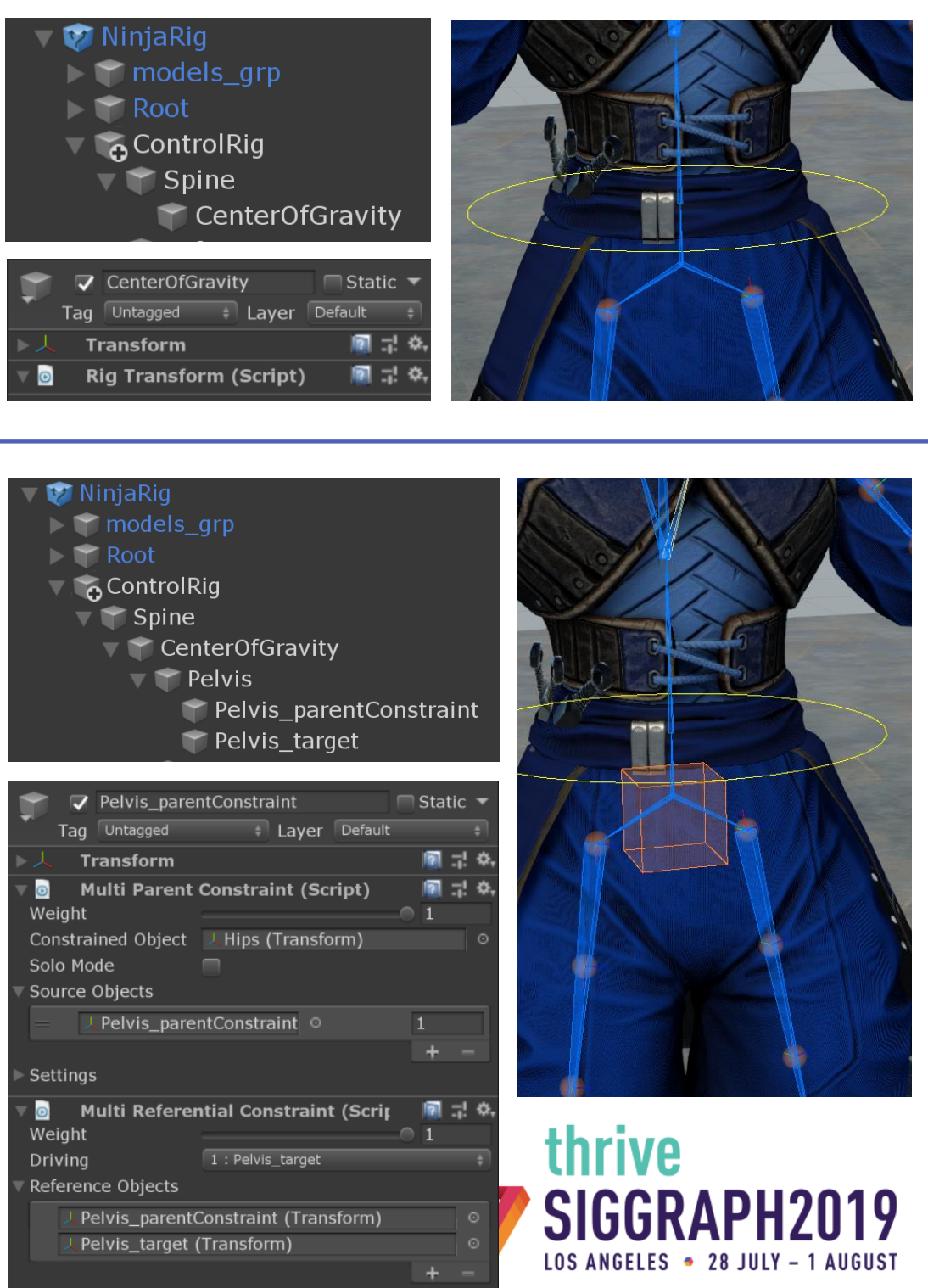

thrive

SIGGRAPH2019 


\section{SETTING UP THE SPINE REGION - TORSO AND HEAD}

Torso

- Setup instructions

1. Create a new GameObject for the Torso (child of CenterOfGravity)

2. Add a TwistChain constraint

3. Create two child GameObjects for Spine_target and Chest_target

1. Align them to the Spine and UpperChest bones

2. Customize the Effector display options

3. Assign the TwistChain Root: Spine_target and Tip: Chest_target

- Neck and Head

- Setup instructions

1. Create a new GameObject for the Neck (child of CenterOfGravity)

2. Add a TwistChain constraint

3. Create a child GameObject for the Head

1. Align it to the Head bone

2. Customize the Effector display options

3. Assign the TwistChain Root: Chest_target and Tip: Head_target
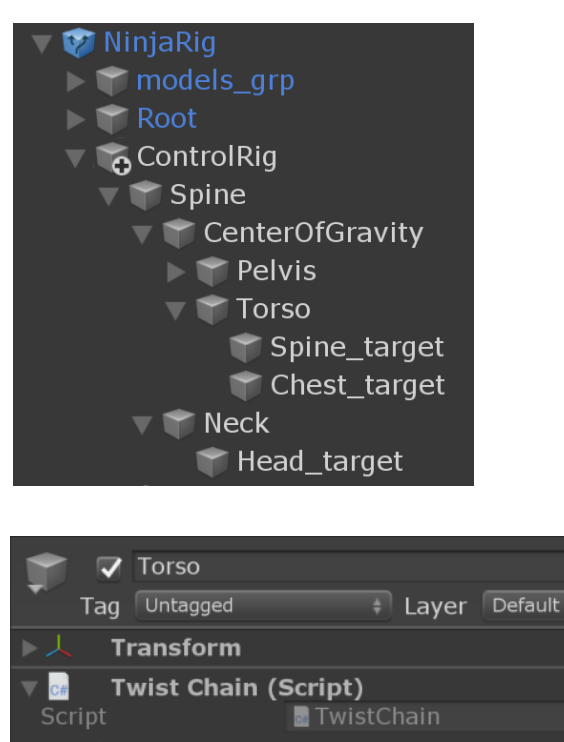

Weight

Weight
Rota
Root

Root
Tip

Root Target

Tip Target

Curve

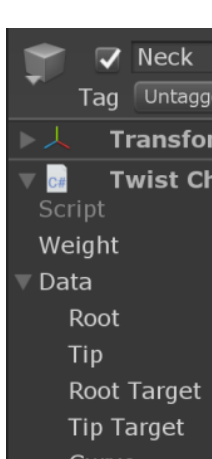

$\nabla$ Neck
Tag Untag
Transform
Twist Cha
eigt
Rata
Root
Tip
Root Targe
Tip Target
Curve

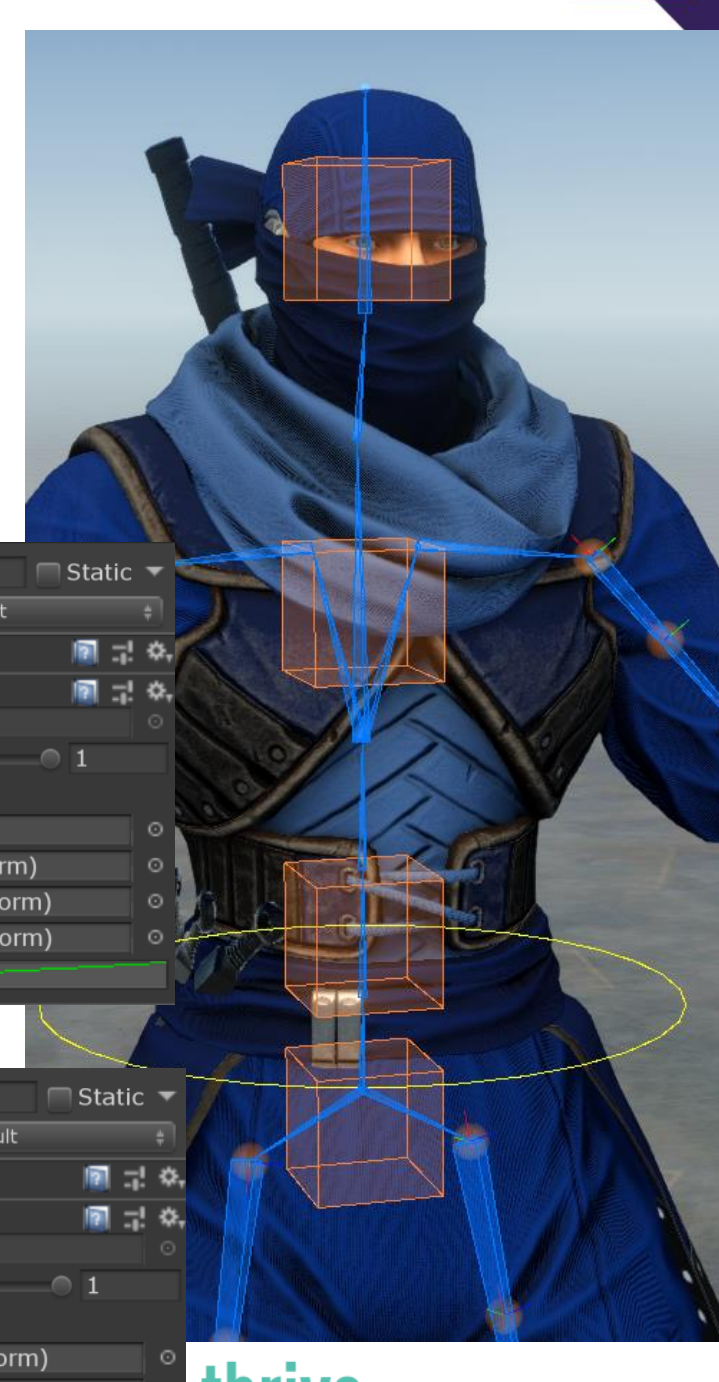

thrive

SIGGRAPH2019 


\section{SETTING UP THE DEFORM RIG}

\section{- Deform Rig}

- Deform rig = bones that move automatically in relation to other bones in the skeleton

- Used to create better looking deformations

- Twist Correction constraints

- On the complete ninja rig observe the DeformRig

- Assets/AnimationRiggingWorkshop/NinjaRigPrefabs/NinjaRig_05_Complete

- Twist Correction constraints are setup for arms and legs

- Select RightUpperArmTwist to see the constraint Inspector

- Note: only one instance of a given rig constraint is allowed per GameObject - this is so that they can be animated
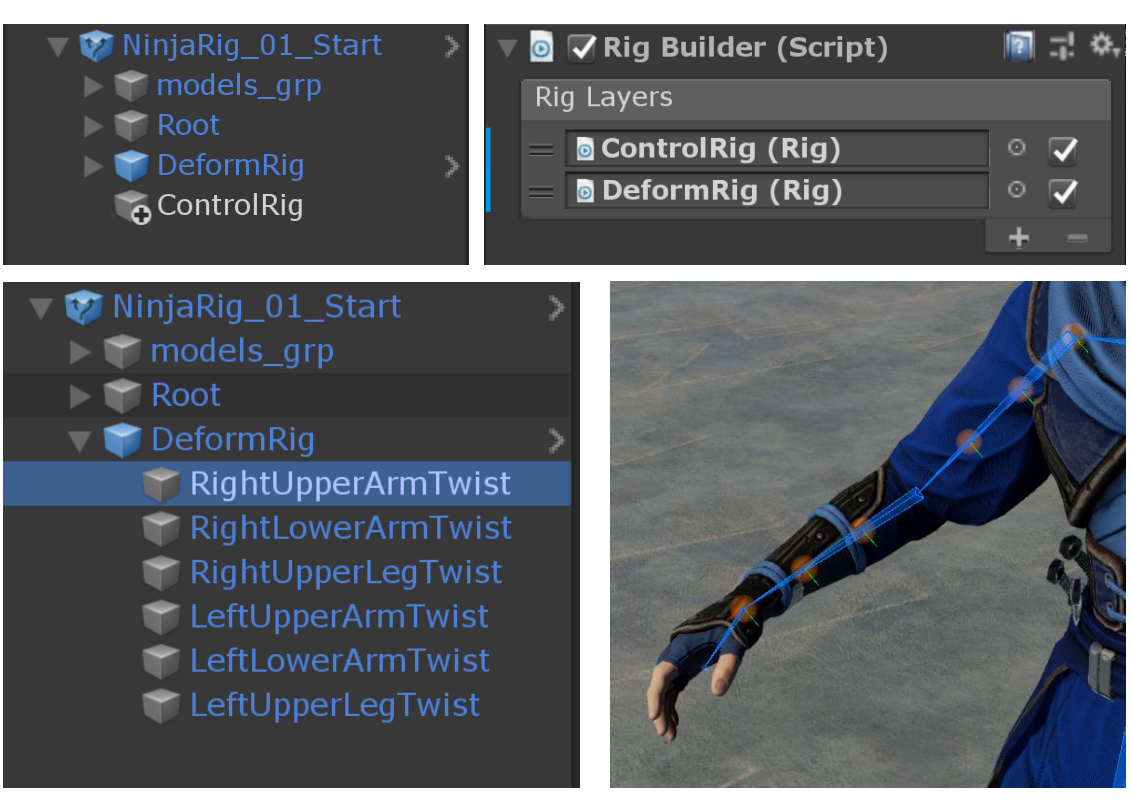

$\nabla$ T Twist Correction (Script)

Weight

Source

Twist Axis

$\nabla$ Twist Nodes

RightUpperArmTwist1 (Transform)

RightUpperArmTwist2 (Transform)

RightUpperArmTwist3 (Transform) 


\section{SETTING UP A SPINE OVERRIDE RIG}

- Spine override rig

- Enables animation to play with an offset. (can be static or animated)

- Example: NinjaRig_06_Override

\section{- Setup instructions}

- Create a duplicate hierarchy for the spine

- Hips $\rightarrow$ Hips_override, etc.

- Add a Bone Renderer to the override bones

- Set all rig constraints drive the override bones

- Add Override Transform constraints to the override bones and set them to drive the actual spine bones
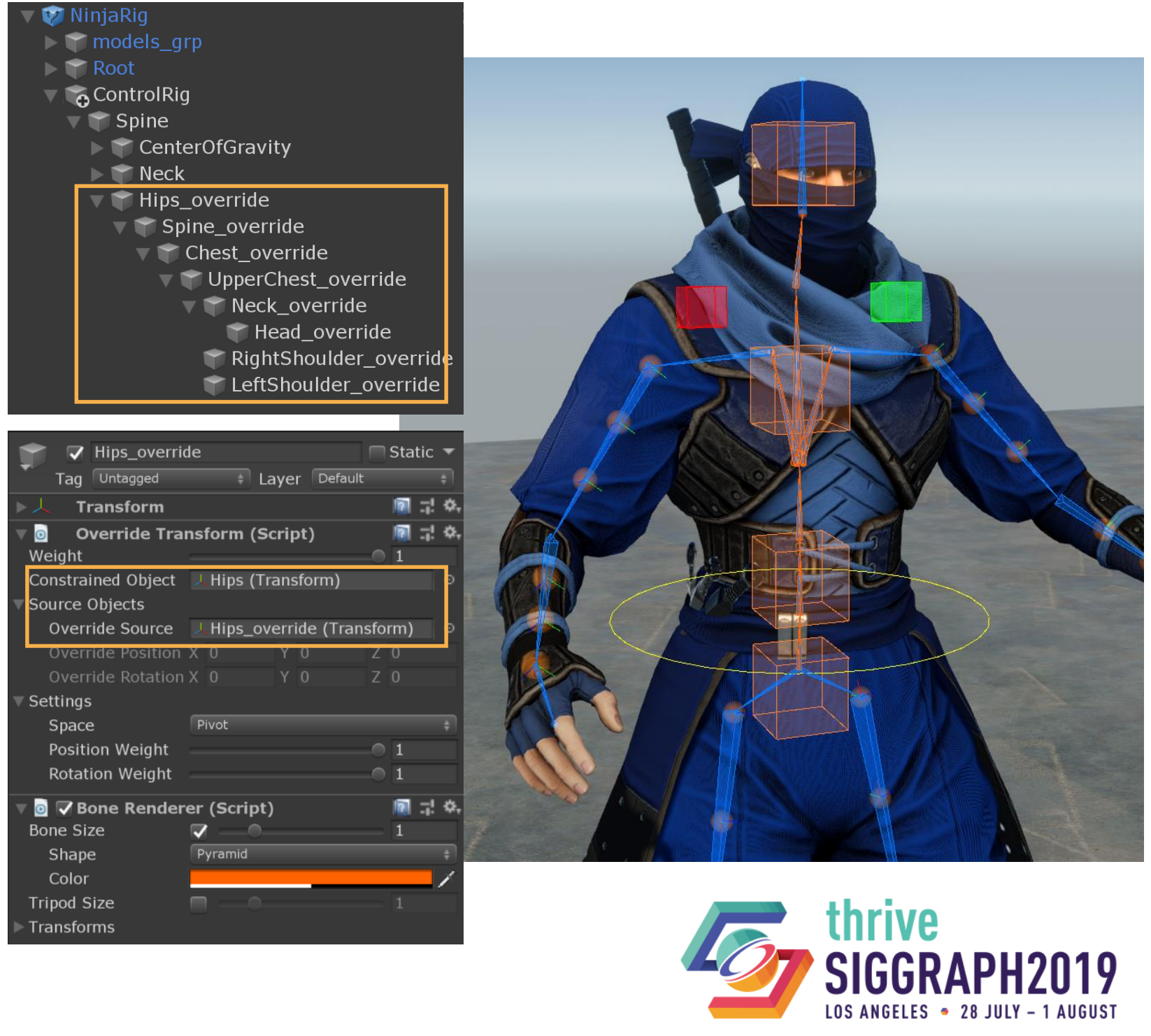


\section{NINJA MEGACITY SCENE}

Open the scene: NinjaMegacityDemo

- One animation clip $\rightarrow$ many rig variations

- Animated rig overrides

- Interact with the rig in Play mode

- Set keyframes in Animation Window Preview mode
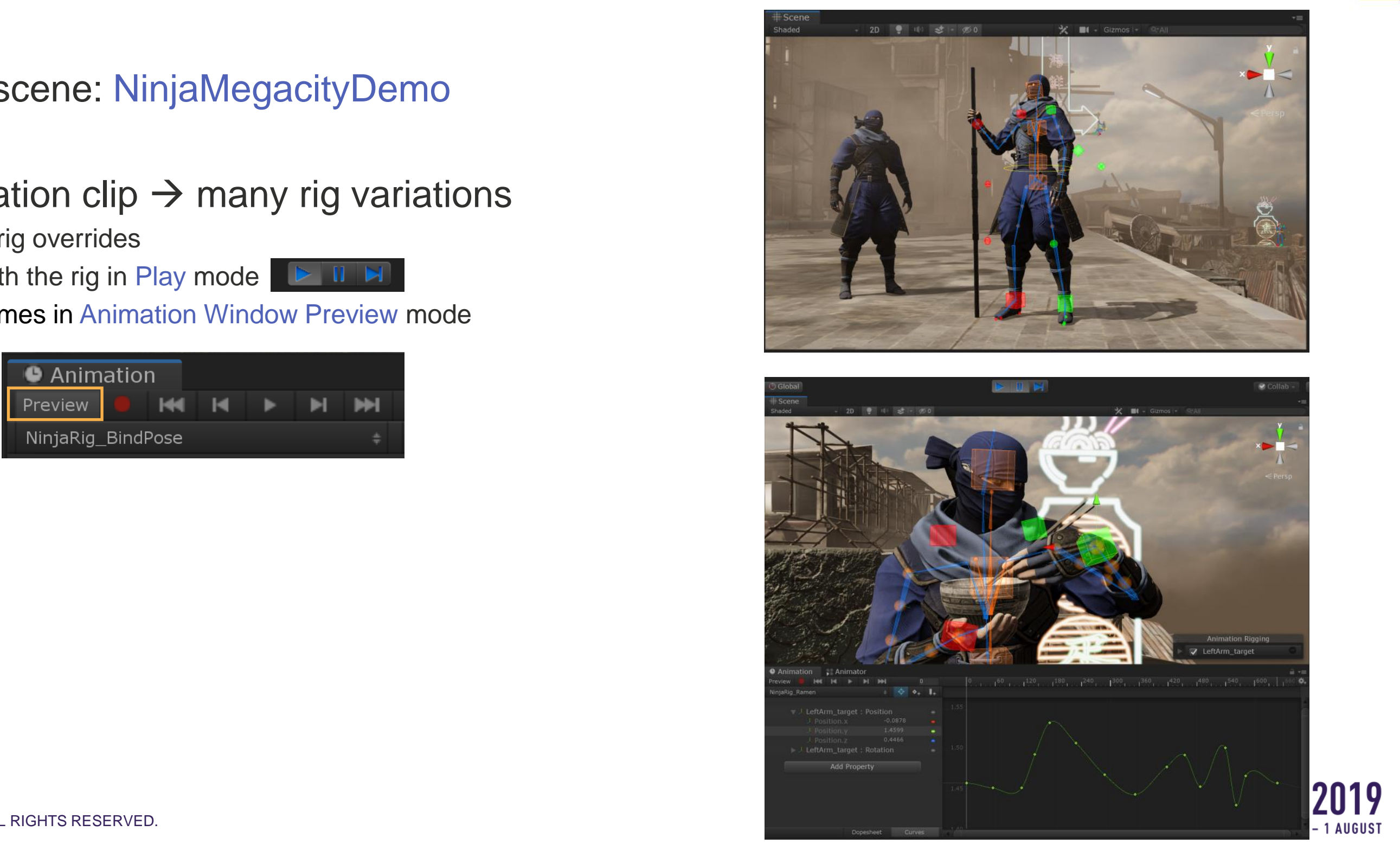
- Extending the Animation Rigging Package with C\# @ 4:00pm

\section{THANKS!}

We look forward to hearing your feedback on Animation Rigging

https://forum.unity.com/forums/animation-previews.141/

Dave Hunt, Technical Artist, davehunt@unity3d.com

Simon Bouvier-Zappa, Animation Developer, simonbz@unity3d.com

Olivier Dionne, Animation Developer, olivierd@unity3d.com

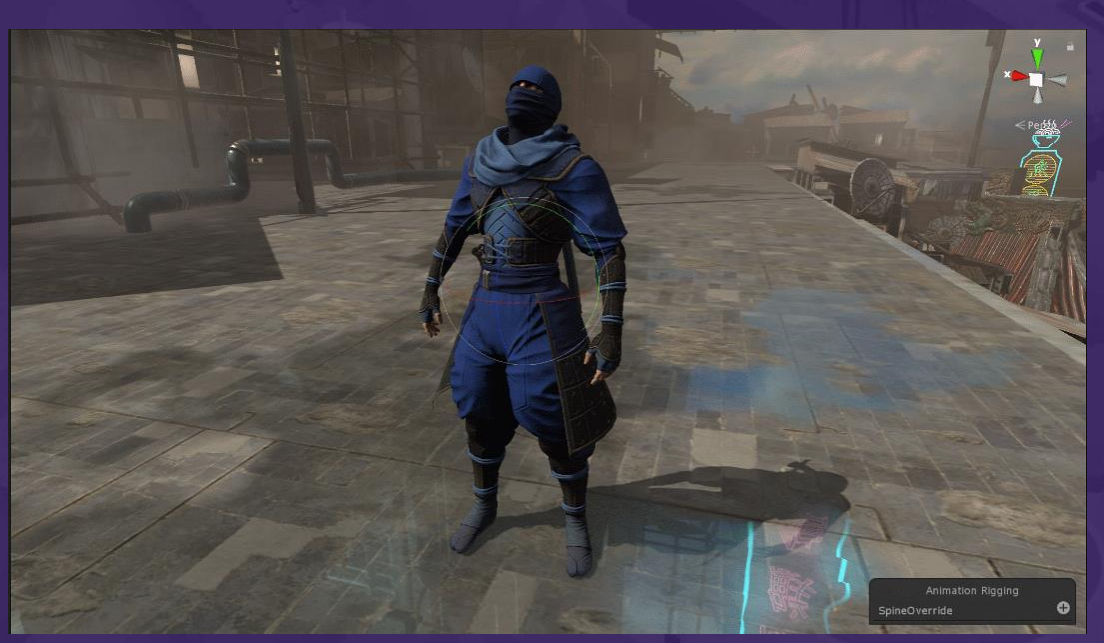

